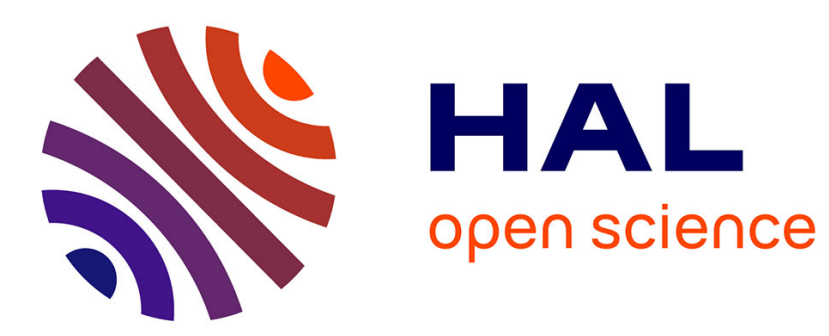

\title{
Uniform controllability of a Stokes problem with a transport term in the zero-diffusion limit
}

\author{
Jon Asier Bárcena-Petisco
}

\section{To cite this version:}

Jon Asier Bárcena-Petisco. Uniform controllability of a Stokes problem with a transport term in the zero-diffusion limit. 2020. hal-02077608v3

\section{HAL Id: hal-02077608 \\ https://hal.science/hal-02077608v3}

Preprint submitted on 7 Apr 2020

HAL is a multi-disciplinary open access archive for the deposit and dissemination of scientific research documents, whether they are published or not. The documents may come from teaching and research institutions in France or abroad, or from public or private research centers.
L'archive ouverte pluridisciplinaire HAL, est destinée au dépôt et à la diffusion de documents scientifiques de niveau recherche, publiés ou non, émanant des établissements d'enseignement et de recherche français ou étrangers, des laboratoires publics ou privés. 


\title{
Uniform controllability of a Stokes problem with a transport term in the zero-diffusion limit
}

\author{
Jon Asier Bárcena-Petisco*†
}

April 7, 2020

\section{Abstract and basic information}

In this paper we consider a Stokes system with Navier-slip boundary conditions. The main results concern the behaviour of the cost of null controllability with respect to the diffusion coefficient when the control acts in the interior. In particular, we prove in $(0, \pi)^{2}$ that for a sufficiently large time the cost decays exponentially as the diffusion coefficient vanishes, whereas in $(0, \pi)^{3}$ we prove that for most of the control domains and for any time $T>0$ the cost explodes exponentially as the diffusion coefficient vanishes.

Key words: Singular limits, spectral decomposition, Stokes system, transport equation, uniform controllability

AMS subject classification: 35B25, 35P10, 93B05, 93C20

Abbreviated title: A transport-diffusion Stokes control problem

Acknowledgements: I would like to thank my thesis advisor Sergio Guerrero and one anonymous reviewer for their multiple remarks. This work has been partially supported by the ANR research project IFSMACS (ANR-15-CE40-0010). This work was supported by grants from Région Ile-de-France.

\footnotetext{
*E-mail: barcena@ljll.math.upmc.fr

${ }^{\dagger}$ This work was done in the Sorbonne Université, Laboratoire Jacques-Louis Lions, F-75005, Paris
} 


\section{Introduction}

\subsection{Main results}

Throughout the paper we are interested in understanding the following control problem:

$$
\begin{cases}y_{t}-\varepsilon \Delta y+\partial_{x_{d}} y+\nabla q=f 1_{\omega} & \text { in } Q \\ \nabla \cdot y=0 & \text { in } Q \\ y \cdot n=0, \quad(D y \cdot n)_{\mathrm{tg}}=0 & \text { on } \Sigma \\ y(0, \cdot)=y^{0} & \text { on } \Omega .\end{cases}
$$

As usual, here and throughout the paper, $T>0, \Omega \subset \mathbb{R}^{d}$ and $\omega \subset \Omega$ are domains, $Q:=(0, T) \times \Omega$, $Q_{\omega}:=(0, T) \times \omega, \Sigma:=(0, T) \times \partial \Omega, D u:=\frac{1}{2}\left(\partial_{x_{i}} u_{j}+\partial_{x_{j}} u_{i}\right)_{i, j}$ for all $u \in \mathbf{L}^{2}(\Omega)$ (in the distributional sense), $n$ denotes the normal vector pointing outwards $\partial \Omega$, " $\partial_{n}$ " denotes the normal outward partial derivative on $\partial \Omega$, and $v_{\mathrm{tg}}:=v-(v \cdot n) n$. The bold notation is used to denote vectorial spaces. As for initial data, $y^{0}$, we take it in $\mathcal{H}(\Omega)$, which is defined by:

$$
\mathcal{H}(\Omega):=\left\{u \in \mathbf{L}^{2}(\Omega): \nabla \cdot u=0 \text { in } \Omega \text { and } u \cdot n=0 \text { on } \partial \Omega\right\} .
$$

We recall that we can define a normal trace which belongs to $H^{-1 / 2}(\partial \Omega)$ in the (closed) subspace of $\mathbf{L}^{2}(\Omega)$ of functions that have a divergence in $L^{2}(\Omega)$. As for the control force, $f$, we want it at least in $\mathbf{L}^{2}\left(Q_{\omega}\right)$ and, if possible, of null first component. Finally, throughout the document $\Omega_{d}:=(0, \pi)^{d}$.

Let us denote the sets of admissible controls as:

$$
\left\{\begin{array}{l}
S_{1}\left(y^{0}\right):=\left\{f \in \mathbf{L}^{2}\left(Q_{\omega}\right): \Phi_{2}\left(y^{0}, f\right)(T, \cdot)=0\right\} \\
S_{2}\left(y^{0}\right):=\left\{f_{2} \in L^{2}\left(Q_{\omega}\right): \Phi_{2}\left(y^{0},\left(0, f_{2}\right)\right)(T, \cdot)=0\right\} \\
S_{3}\left(y^{0}\right):=\left\{f \in \mathbf{L}^{2}\left(Q_{\omega}\right): \Phi_{3}\left(y^{0}, f\right)(T, \cdot)=0\right\}
\end{array}\right.
$$

for $y^{0} \in \mathcal{H}\left(\Omega_{d}\right)$, for $f \in \mathbf{L}^{2}\left(Q_{\omega}\right)$ and for $\Phi_{d}\left(y^{0}, f\right)$ the solution of $\left[1.1\right.$ in $\Omega_{d}$. Thanks to [18], we know that $S_{1}$ and $S_{3}$ are non-empty, whereas we obtain in Section 3.1 that $S_{2}$ is non-empty by proving the corresponding observability estimate. As for the cost of the control, we measure it with the usual norms. In fact, we denote:

$$
\left\{\begin{array}{l}
K_{1}(T, \varepsilon, \omega):=\sup _{y^{0} \in \mathcal{H}\left(\Omega_{2}\right) \backslash\{0\}} \inf _{f \in S_{1}\left(y^{0}\right)} \frac{\|f\|_{\mathbf{L}^{2}\left(Q_{\omega}\right)}}{\left\|y^{0}\right\|_{\mathbf{L}^{2}\left(\Omega_{2}\right)}} \\
K_{2}(T, \varepsilon, \omega):=\sup _{y^{0} \in \mathcal{H}\left(\Omega_{2}\right) \backslash\{0\}} \inf _{f_{2} \in S_{2}\left(y^{0}\right)} \frac{\left\|f_{2}\right\|_{L^{2}\left(Q_{\omega}\right)}}{\left\|y^{0}\right\|_{\mathbf{L}^{2}\left(\Omega_{2}\right)}} \\
K_{3}(T, \varepsilon, \omega):=\sup _{y^{0} \in \mathcal{H}\left(\Omega_{3}\right) \backslash\{0\}} \inf _{f \in S_{3}\left(y^{0}\right)} \frac{\|f\|_{\mathbf{L}^{2}\left(Q_{\omega}\right)}}{\left\|y^{0}\right\|_{\mathbf{L}^{2}\left(\Omega_{3}\right)}}
\end{array}\right.
$$

The main results that we prove in the paper are the following ones:

Theorem 1.1. We have the following results for the control system 1.1):

1. Let $\omega \subset \Omega_{2}$ a domain. Then, there are some $c, C, T_{0}>0$ such that, if $T>T_{0}$ and $\varepsilon \in(0,1)$ :

$$
K_{2}(T, \varepsilon, \omega) \leq C e^{-c \varepsilon^{-1}} .
$$


2. Let $h \in(0, \pi)$ and $\omega \subset(0, \pi) \times(\pi-h, \pi)$ a domain. Then, for any $T \in(0,2(\pi-h))$, there is $c>0$ such that, if $\varepsilon \in(0,1)$ :

$$
K_{1}(T, \varepsilon, \omega) \geq c e^{c \varepsilon^{-1}}
$$

3. Let $h \in(0, \pi)$ and $\omega \subset(0, \pi) \times(0, h)$ a domain. Then, for any $T \in(0, \pi-h)$, there is $c>0$ such that, if $\varepsilon \in(0,1)$ :

$$
K_{1}(T, \varepsilon, \omega) \geq c e^{c \varepsilon^{-1}}
$$

4. Let $h \in(0, \pi)$ and $\omega \subset(0, \pi)^{2} \times(\pi-h, \pi)$ a domain. Then, for any $T>0$ there is $c>0$ such that, if $\varepsilon \in(0,1)$ :

$$
K_{3}(T, \varepsilon, \omega) \geq c e^{c \varepsilon^{-1}}
$$

Remark 1.2. The results in dimension 2 are not surprising as they are similar to those in $9,20,14,25,26$. The result in dimension 3 (see Item 4), however, is surprising because it holds for all $T>0$ and for a wide range of control domains (in particular, for all the control domains compactly supported in $\Omega_{3}$ ). This result is explained because we have much more freedom to construct eigenfunctions of the adjoint operator (see 1.5 and Remark 4.1 in Section 4 below). Indeed, we can construct in dimension 3 a continuous family of eigenfunctions whose respective eigenvalues do not explode when $\varepsilon \rightarrow 0^{+}$. The existence of a system with this property is a novelty with respect to the existing literature.

Remark 1.3. The analogous results to the ones stated in Theorem 1.1 and in the lemmas and propositions throughout the paper are true for any rectangle or cuboids and with any velocity parallel to their edges. Indeed, all the operations can be replicated under those assumptions. The reason of restricting to $(0, \pi)^{2}$ or $(0, \pi)^{3}$ is to reduce the number of operations so that the reader can focus on the main ideas of the proof.

In order to estimate the cost of the control, we study the adjoint system:

$$
\begin{cases}-\varphi_{t}-\varepsilon \Delta \varphi-\partial_{x_{d}} \varphi+\nabla p=0 & \text { in } Q, \\ \nabla \cdot \varphi=0 & \text { in } Q, \\ \varphi \cdot n=0, \quad\left(2 \varepsilon D \varphi \cdot n+\varphi n_{d}\right)_{\mathrm{tg}}=0 & \text { on } \Sigma, \\ \varphi(T, \cdot)=\varphi^{T} & \text { on } \Omega,\end{cases}
$$

for $d$ the dimension of $\Omega$. We recall the following classical result (see, for instance, [27, 23]):

$$
\begin{cases}{\left[K_{1}(T, \varepsilon, \omega)\right]^{2}=} & \sup _{\varphi^{T} \in \mathcal{H}\left(\Omega_{2}\right) \backslash\{0\}} \frac{\int_{\Omega_{2}}|\varphi(0, x)|^{2} d x}{\iint_{Q_{\omega}}|\varphi|^{2} d x d t}, \\ {\left[K_{2}(T, \varepsilon, \omega)\right]^{2}=} & \sup _{\varphi^{T} \in \mathcal{H}\left(\Omega_{2}\right) \backslash\{0\}} \frac{\int_{\Omega_{2}}|\varphi(0, x)|^{2} d x}{\iint_{Q_{\omega}}\left|\varphi_{2}\right|^{2} d x d t}, \\ {\left[K_{3}(T, \varepsilon, \omega)\right]^{2}=\sup _{\varphi^{T} \in \mathcal{H}\left(\Omega_{3}\right) \backslash\{0\}} \frac{\int_{\Omega_{3}}|\varphi(0, x)|^{2} d x}{\iint_{Q_{\omega}}|\varphi|^{2} d x d t} .}\end{cases}
$$

While working in $\Omega_{2}$ we use the following notation:

$$
\Sigma_{l r}:=(0, T) \times\{0, \pi\} \times(0, \pi), \text { and } \Sigma_{b t}:=(0, T) \times(0, \pi) \times\{0, \pi\} .
$$


In order to understand the (energy) solutions of (1.3), we do a spectral decomposition. In particular, we look for the solutions of:

$$
\begin{cases}-\varepsilon \Delta u-\partial_{x_{d}} u+\nabla p=\lambda u & \text { in } \Omega \\ \nabla \cdot u=0 & \text { in } \Omega \\ u \cdot n=0, \quad\left(2 \varepsilon D u \cdot n+u n_{d}\right)_{\mathrm{tg}}=0 & \text { on } \partial \Omega\end{cases}
$$

for $d$ the dimension of $\Omega$.

Remark 1.4. One interesting property of system 1.3 considered in $\Omega_{2}$ is that, as long as $\varphi^{T} \in \mathcal{H}\left(\Omega_{2}\right)$, the gradient of the pressure of the solution of 1.3 is null for any given time. Indeed, by taking the divergence in 1.3$)_{1}$ we obtain that $-\Delta p=0$, so the result is proved if we show that $\partial_{n} p=0$ on $\Sigma$. For that purpose, we multiply $(1.3)_{1}$ by $n$. First, $\varphi_{t} \cdot n=0$ as $\varphi \cdot n=0$. Second, we have that:

$$
-\left(\varepsilon \Delta \varphi+\partial_{x_{2}} \varphi\right) \cdot n 1_{\Sigma_{l r}}=-\left(\varepsilon \partial_{x_{1}}^{2} \varphi_{1}+\varepsilon \partial_{x_{2}}^{2} \varphi_{1}+\partial_{x_{2}} \varphi_{1}\right) n_{1} 1_{\Sigma_{l r}}=\varepsilon \partial_{x_{2}} \partial_{x_{1}} \varphi_{2} n_{1} 1_{\Sigma_{l r}}=0 .
$$

We have used on the previous equality that $n_{2}=0$ on $\left.\Sigma_{l r}, 1.3\right]_{2}$ and that $\varphi_{1}=\partial_{x_{1}} \varphi_{2}=0$ on $\Sigma_{l r}$ because of 1.3$]_{3}$. And finally, we have that:

$$
-\left(\varepsilon \Delta \varphi+\partial_{x_{2}} \varphi\right) \cdot n 1_{\Sigma_{b t}}=-\left(\varepsilon \partial_{x_{1}}^{2} \varphi_{2}+\varepsilon \partial_{x_{2}}^{2} \varphi_{2}+\partial_{x_{2}} \varphi_{2}\right) n_{2} 1_{\Sigma_{b t}}=\left(\varepsilon \partial_{x_{1}} \partial_{x_{2}} \varphi_{1}+\partial_{x_{1}} \varphi_{1}\right) n_{2} 1_{\Sigma_{b t}}=0 .
$$

We have used that $n_{1}=0$ on $\left.\Sigma_{b t}, 1.3\right]_{2}$ and that $\varphi_{2}=\varepsilon \partial_{x_{2}} \varphi_{1}+\varphi_{1}=0$ on $\Sigma_{b t}$ because of 1.3$]_{3}$.

Remark 1.5. An immediate consequence of Remark 1.4 is that if $\varphi^{T} \in \mathcal{H}\left(\Omega_{2}\right), \varphi_{1}$ (the first component of the solution $\varphi$ of $(1.3)$ in $\left.\Omega_{2}\right)$ satisfies:

$$
\begin{cases}-z_{t}-\varepsilon \Delta z-\partial_{x_{2}} z=0 & \text { in } Q, \\ z=0 & \text { on } \Sigma_{l r}, \\ \varepsilon \partial_{x_{2}} z+z=0 & \text { on } \Sigma_{b t}, \\ z(T, \cdot)=z^{T} & \text { on } \Omega_{2},\end{cases}
$$

for $z^{T}=\varphi_{1}^{T}$. In addition, $\varphi_{2}$ satisfies:

$$
\begin{cases}-z_{t}-\varepsilon \Delta z-\partial_{x_{2}} z=0 & \text { in } Q, \\ \partial_{x_{1}} z=\partial_{n} z=0 & \text { on } \Sigma_{l r}, \\ z=0 & \text { on } \Sigma_{b t}, \\ z(T, \cdot)=z^{T} & \text { on } \Omega_{2},\end{cases}
$$

for $z^{T}=\varphi_{2}^{T}$.

Throughout the paper we use $c$ and $C$ to denote strictly positive constants, which might be different each time. We denote the small constants by $c$ and the large ones by $C$.

\section{$1.2 \quad$ Historical background}

The first control system with a small diffusion and a transport term that was analysed was the heat equation in dimension 1 with Dirichlet boundary conditions in [9. Afterwards, the same problem but 
in any dimension and with any speed belonging to $W^{1,+\infty}\left(\mathbb{R}^{+} \times \Omega\right)$ was studied in [20]. More recently, better approximations of the optimal time in which the cost of the control decays have been given: the upper bound was improved in [14, 25] (in the first one through complex analysis and in the second one by transforming the original equation into the pure heat equation), and the lower bound was improved in [26] through complex analysis and properties of the entire functions. As for similar results, work has been done in the the Burgers equation (see [15), in the KdV equation (see [16, 17, 2, 3]) and in an artificial advection-diffusion problem (see [5, 6]). As for the Stokes system with small diffusion and a transport term, this is the first time that such a system has been studied and is, indeed, one of the contributions of the paper.

The study of control problems associated to Stokes systems with Navier-slip boundary condition (see (1.1) ) is not new in the literature: the existence of $d$ dimensional controls leading to null controllability was proved in [18, while the existence of $d-1$ dimensional controls has recently been proved in [21]. The need of working in a specific domain to simplify the problem is not new either. For example, recently, in [10, they prove that the Navier-Stokes is (globally) null controllable in any rectangle with boundary controls at two opposing edges and with a phantom force (see also [19] for a weaker result in cuboids).

Finally, the idea of using spectral decomposition is not new in Control Theory (see, for instance, [8]). Indeed, for the heat and the Stokes context alone, there are many documents which deal with eigenfunctions of the elliptic operator, for proving the existence of some control (see, for example, 22, 24, 4]), for estimating the cost of the control (see, for instance, [1]), and for giving negative answer to the existence of a control (see, for example, 24]). As for a system with small diffusion and a transport term, a spectral decomposition indirectly appears in [9, 26], when getting lower bounds for the optimal time $T_{0}$ in which the cost of the control decays exponentially with $\varepsilon$. However, as far as the author knows, this is the first time that a spectral decomposition has been used to get the dissipation estimate in a transport-diffusion system.

The rest of the paper is organized as follows: in Section 2 we study systems (1.3) and 1.5 for $\Omega_{2}$, in Section 3 we study the cost of the control for $\Omega_{2}$, in Section 4 we study the control problem (1.1) and its adjoint system for $\Omega_{3}$, in Section 5 we do some further comments about the techniques and point out some open problems, and in Appendix $\mathrm{A}$ we prove a technical result.

\section{Spectral decomposition of $\mathcal{H}\left((0, \pi)^{2}\right)$ and some immediate con- sequences}

In this section we look for solutions of 1.5 in $\Omega_{2}$ and use them to extract information about the solutions of 1.3 in $\Omega_{2}$. In particular, in Section 2.1 we provide the proof of a technical result, in Section 2.2 we get some eigenfunctions (solutions of 1.5 ) which form a total set in $\mathcal{H}\left(\Omega_{2}\right)$, and in Section 2.3 we use those eigenfunctions to extract information about the solutions of $(1.3)$. We avoid using Remark 
1.4 and Remark 1.5 to have a proof that can be generalized to other Stokes systems and because it does not spare us many calculations.

As for the notation, given any real Banach space $(V,\|\cdot\|)$ and $S \subset V$, we recall that $\operatorname{span}(S)$ denotes the set of all linear combinations of the elements of $S$. Moreover, we recall that $S$ is a total set if $\overline{\operatorname{span}(S)}=V$. Also,

$$
l^{2}:=\left\{\left(a_{k}\right)_{k \in \mathbb{N}_{*}}: a_{k} \in \mathbb{R} \text { and } \sum_{k \in \mathbb{N}_{*}} a_{k}^{2}<+\infty\right\} .
$$

Besides, for any bounded domain $\Theta \subset \mathbb{R}^{d}$, we denote:

$$
L_{0}^{2}(\Theta):=\left\{g \in L^{2}(\Theta): \int_{\Theta} g(x) d x=0\right\} .
$$

Moreover, we use $m=\left(m_{1}, m_{2}\right) \in\left(\mathbb{N}_{*}\right)^{2}$ to index the eigenfunctions. Finally, we denote the set of functions with separated variables having null normal trace and null divergence as:

$$
S V\left(\Omega_{2}\right):=\left\{\left(\int_{0}^{x_{1}} g_{1}(s) d s g_{2}\left(x_{2}\right),-g_{1}\left(x_{1}\right) \int_{0}^{x_{2}} g_{2}(s) d s\right): g_{1}, g_{2} \in L_{0}^{2}(0, \pi)\right\} .
$$

\subsection{Some functional analysis results}

In order to look for eigenfunctions, we look among those which have a simple structure; in particular, among those which belong to $S V\left(\Omega_{2}\right)$. Indeed, by construction, $S V\left(\Omega_{2}\right) \subset \mathcal{H}\left(\Omega_{2}\right)$. Moreover, the interest of $S V\left(\Omega_{2}\right)$ is that it is a total set:

Proposition 2.1. We have:

$$
\overline{\operatorname{span}\left(S V\left(\Omega_{2}\right)\right)}=\mathcal{H}\left(\Omega_{2}\right) .
$$

Remark 2.2. This result is not surprising since it is well-known that functions with separated variables form a total set in $L^{2}\left(\Omega_{2}\right)$.

In order to prove Proposition 2.1, we first need some technical results whose proofs, up to the author's knowledge, do not appear explicitly in any book or paper:

Lemma 2.3. Let $A_{1}, A_{2}, B_{1}, B_{2} \in \mathbb{R}$. Let $\Theta:=\left(A_{1}, A_{2}\right) \times\left(B_{1}, B_{2}\right), p \in(1,+\infty)$ and $h \in L^{p}(\Theta)$ a function such that:

$$
\int_{\Theta} h\left(x_{1}, x_{2}\right) g_{1}\left(x_{1}\right) g_{2}\left(x_{2}\right) d x=0,
$$

for all $g_{1} \in L_{0}^{\infty}\left(A_{1}, A_{2}\right)$ and for all $g_{2} \in L_{0}^{\infty}\left(B_{1}, B_{2}\right)$. Then, there are $h_{1} \in L^{p}\left(A_{1}, A_{2}\right)$ and $h_{2} \in L^{p}\left(B_{1}, B_{2}\right)$ such that:

$$
h\left(x_{1}, x_{2}\right)=h_{1}\left(x_{1}\right)+h_{2}\left(x_{2}\right) \text { almost everywhere in } \Theta \text {, }
$$

and such that:

$$
\int_{A_{1}}^{A_{2}} h_{1}(s) d s=0 .
$$

For the proof, we follow the classical scheme of first proving a regular version of the lemma, and then generalizing it to weaker spaces thanks to mollifiers. 
Lemma 2.4. Let $A_{1}, A_{2}, B_{1}, B_{2} \in \mathbb{R}$. Let $\Theta:=\left(A_{1}, A_{2}\right) \times\left(B_{1}, B_{2}\right), N \geq 0$ and $h \in C^{N}(\bar{\Theta})$ a function such that:

$$
\int_{\Theta} h\left(x_{1}, x_{2}\right) g_{1}\left(x_{1}\right) g_{2}\left(x_{2}\right) d x=0,
$$

for all $g_{1} \in L_{0}^{\infty}\left(A_{1}, A_{2}\right)$ and for all $g_{2} \in L_{0}^{\infty}\left(B_{1}, B_{2}\right)$. Then, there are $h_{1} \in C^{N}\left(\left[A_{1}, A_{2}\right]\right)$ and $h_{2} \in$ $C^{N}\left(\left[B_{1}, B_{2}\right]\right)$ such that:

$$
h\left(x_{1}, x_{2}\right)=h_{1}\left(x_{1}\right)+h_{2}\left(x_{2}\right) \text { in } \bar{\Theta},
$$

and such that:

$$
\int_{A_{1}}^{A_{2}} h_{1}(s) d s=0
$$

We remark that 2.3 and 2.5) are required to have some continuity in the decomposition $\left(h_{1}, h_{2}\right)$. Moreover, they also provide uniqueness.

Proof of Lemma 2.4. Since the Lebesgue measure works well with translations and dilations, it suffices to prove the result for $\Theta=(0,1)^{2}$. In particular, it suffices to prove that for all $x_{1}, x_{2}, \tilde{x}_{1}, \tilde{x}_{2} \in(0,1)$ such that $x_{1} \neq \tilde{x}_{1}$ and $x_{2} \neq \tilde{x}_{2}$ :

$$
h\left(x_{1}, x_{2}\right)+h\left(\tilde{x}_{1}, \tilde{x}_{2}\right)=h\left(x_{1}, \tilde{x}_{2}\right)+h\left(\tilde{x}_{1}, x_{2}\right) .
$$

Indeed, by continuity, 2.6 extends to all $x_{1}, x_{2}, \tilde{x}_{1}, \tilde{x}_{2} \in[0,1]$. Consequently, if we consider $\left(\tilde{x}_{1}, \tilde{x}_{2}\right)=$ $(0,0)$, we get that:

$$
h\left(x_{1}, x_{2}\right)=h\left(x_{1}, 0\right)+h\left(0, x_{2}\right)-h(0,0) .
$$

Let us suppose that there exist $a_{1}, a_{2}, b_{1}, b_{2} \in(0,1)$ such that $a_{1} \neq a_{2}$ and $b_{1} \neq b_{2}$ and such that 2.6 is not true. By symmetry, we can suppose that:

$$
h\left(a_{1}, b_{1}\right)+h\left(a_{2}, b_{2}\right)>h\left(a_{1}, b_{2}\right)+h\left(a_{2}, b_{1}\right) .
$$

Then, for some $\delta>0$ small enough (in particular smaller than $a_{1}, a_{2}, b_{1}, b_{2}, 1-a_{1}, 1-a_{2}, 1-b_{1}, 1-b_{2}$ ), by the continuity of $h$, we have for all $s=\left(s_{1}, s_{2}\right) \in[-\delta, \delta]^{2}$ the inequality:

$$
h\left(a_{1}+s_{1}, b_{1}+s_{2}\right)+h\left(a_{2}+s_{1}, b_{2}+s_{2}\right)>h\left(a_{1}+s_{1}, b_{2}+s_{2}\right)+h\left(a_{2}+s_{1}, b_{1}+s_{2}\right) .
$$

Let us define:

$$
g_{1}:=1_{\left(a_{1}-\delta, a_{1}+\delta\right)}-1_{\left(a_{2}-\delta, a_{2}+\delta\right)},
$$

and:

$$
g_{2}:=1_{\left(b_{1}-\delta, b_{1}+\delta\right)}-1_{\left(b_{2}-\delta, b_{2}+\delta\right)} .
$$

Then, we have that $g_{1}, g_{2} \in L_{0}^{\infty}(0,1)$, but:

$$
\begin{aligned}
\int_{\Theta} h\left(x_{1}, x_{2}\right) g_{1}\left(x_{1}\right) g_{2}\left(x_{2}\right) d x=\int_{[-\delta, \delta]^{2}}\left(h\left(a_{1}+s_{1}, b_{1}+s_{2}\right)+h\left(a_{2}+s_{1}, b_{2}+s_{2}\right)\right. & \\
& \left.-h\left(a_{1}+s_{1}, b_{2}+s_{2}\right)-h\left(a_{2}+s_{1}, b_{1}+s_{2}\right)\right) d s_{1} d s_{2}>0
\end{aligned}
$$

which contradicts 2.4). 
For the following proof we use the notation $I_{k}:=\left(\frac{1}{k}, 1-\frac{1}{k}\right)$ and $I_{k}^{2}:=I_{k} \times I_{k}$, for $k$ sufficiently large.

Proof of Lemma 2.3. Again, since the Lebesgue measure works well with translations and dilations, it suffices to prove the result for $\Theta=(0,1)^{2}$. In particular, in order to prove the existence of such $h_{1}, h_{2}$, it suffices to prove that, for all $k \in \mathbb{N}_{*}$, there is $h_{1, k} \in L_{0}^{p}\left(I_{k}\right)$ and $h_{2, k} \in L^{p}\left(I_{k}\right)$ with $\left\|h_{1, k}\right\|_{L^{p}\left(I_{k}\right)}$ and $\left\|h_{2, k}\right\|_{L^{p}\left(I_{k}\right)}$ bounded uniformly, such that:

$$
h\left(x_{1}, x_{2}\right)=h_{1, k}\left(x_{1}\right)+h_{2, k}\left(x_{2}\right) \text { almost everywhere in } I_{k}^{2} .
$$

Indeed, we just have to take weak limits (up to extracting a subsequence) at both sides of:

$$
h\left(x_{1}, x_{2}\right) 1_{I_{k}^{2}}\left(x_{1}, x_{2}\right)=h_{1, k}\left(x_{1}\right) 1_{I_{k}}\left(x_{1}\right)+h_{2, k}\left(x_{2}\right) 1_{I_{k}}\left(x_{2}\right),
$$

and use that the unit ball of $L^{p}(0,1)$ is weakly compact.

In order to prove the existence of $h_{1, k}$ and $h_{2, k}$, let us consider $\xi$ an even mollifier (a positive $C^{\infty}(\mathbb{R}$ ) function such that $\left.\|\xi\|_{L^{1}(\mathbb{R})}=1\right)$ supported in $[-1,1]$. We denote $\xi_{\varepsilon}(s):=\varepsilon^{-1} \xi\left(\varepsilon^{-1} s\right)$. Let us now consider $g_{1}, g_{2} \in L_{0}^{\infty}\left(I_{k}\right)$ (and null outside $I_{k}$ ). We have, for all $\varepsilon<k^{-1}$, by Fubini: $g_{1} \star \xi_{\varepsilon}, g_{2} \star \xi_{\varepsilon} \in L_{0}^{\infty}(0,1)$. Thus, by (2.2), Fubini, the even symmetry of $\xi$, and the support of $g_{1}$ and $g_{2}$, we have that:

$$
\begin{aligned}
0=\int_{\Theta} h\left(x_{1}, x_{2}\right) & \left(\int_{0}^{1} g_{1}\left(s_{1}\right) \xi_{\varepsilon}\left(x_{1}-s_{1}\right) d s_{1}\right)\left(\int_{0}^{1} g_{2}\left(s_{2}\right) \xi_{\varepsilon}\left(x_{2}-s_{2}\right) d s_{2}\right) d x \\
& =\int_{\Theta} g_{1}\left(s_{1}\right) g_{2}\left(s_{2}\right)\left(\int_{\Theta} h\left(x_{1}, x_{2}\right) \xi_{\varepsilon}\left(s_{1}-x_{1}\right) \xi_{\varepsilon}\left(s_{2}-x_{2}\right) d x\right) d s_{1} d s_{2} \\
& =\int_{I_{k}^{2}} g_{1}\left(s_{1}\right) g_{2}\left(s_{2}\right)\left(h \star\left(\xi_{\varepsilon} \otimes \xi_{\varepsilon}\right)\right)\left(s_{1}, s_{2}\right) d s_{1} d s_{2} .
\end{aligned}
$$

Let us denote $h_{\varepsilon}:=h \star\left(\xi_{\varepsilon} \otimes \xi_{\varepsilon}\right)$. Since $\xi$ is a mollifier, $h_{\varepsilon} \in C^{\infty}\left(\overline{I_{k}^{2}}\right)$ and:

$$
h_{\varepsilon} \rightarrow h \text { in } L^{p}\left(I_{k}^{2}\right)
$$

Moreover, since identity (2.8) is satisfied for any $g_{1}, g_{2}$ in $L_{0}^{\infty}\left(I_{k}\right)$, we have that, because of Lemma 2.4 the existence of $h_{1, \varepsilon}$ and $h_{2, \varepsilon}$ in $C^{\infty}\left(\overline{I_{k}}\right)$ such that:

$$
h_{\varepsilon}\left(x_{1}, x_{2}\right)=h_{1, \varepsilon}\left(x_{1}\right)+h_{2, \varepsilon}\left(x_{2}\right) \text { in } I_{k}^{2},
$$

and:

$$
\int_{I_{k}} h_{1, \varepsilon}(s) d s=0 .
$$

Because of 2.9 and the justifications given in the previous paragraph it suffices to prove that $\left\|h_{1, \varepsilon}\right\|_{L^{p}\left(I_{k}\right)}$ and $\left\|h_{2, \varepsilon}\right\|_{L^{p}\left(I_{k}\right)}$ are uniformly bounded. In the case of $h_{2, \varepsilon}$, using 2.10), we get that:

$$
\left(\frac{k-2}{k}\right) h_{2, \varepsilon}\left(x_{2}\right)=\int_{I_{k}} h_{\varepsilon}\left(x_{1}, x_{2}\right) d x_{1} \text { in } I_{k} .
$$

Thus, by Fubini, Hölder and Young, we get for $k \geq 4$ the estimate:

$$
\left\|h_{2, \varepsilon}\right\|_{L^{p}\left(I_{k}\right)} \leq 2\left\|h_{\varepsilon}\right\|_{L^{p}\left(I_{k}^{2}\right)} \leq 2\|h\|_{L^{p}(\Theta)} .
$$


As for $h_{1, \varepsilon}$, since:

$$
\left(\frac{k-2}{k}\right) h_{1, \varepsilon}\left(x_{1}\right)=\int_{I_{k}}\left(h_{\varepsilon}\left(x_{1}, x_{2}\right)-h_{2, \varepsilon}\left(x_{2}\right)\right) d x_{2},
$$

by the triangular inequality, Fubini, Hölder, Young and (2.11), we get for $k \geq 4$ the estimate:

$$
\left\|h_{1, \varepsilon}\right\|_{L^{p}\left(I_{k}\right)} \leq 6\|h\|_{L^{p}(\Theta)} .
$$

Now we are ready to prove Proposition 2.1

Proof of proposition 2.1. Since $\overline{\operatorname{span}\left(S V\left(\Omega_{2}\right)\right)}$ is a closed vectorial subspace of $\mathcal{H}\left(\Omega_{2}\right)$, it suffices to prove that:

$$
\overline{\operatorname{span}\left(S V\left(\Omega_{2}\right)\right)^{\perp}}=\{0\} .
$$

So, let us consider $u \in \mathcal{H}\left(\Omega_{2}\right)$ such that:

$$
\int_{\Omega_{2}} u \cdot v d x=0
$$

for any $v \in S V\left(\Omega_{2}\right)$. Recalling 2.1 , that $L_{0}^{\infty}(0, \pi) \subset L_{0}^{2}(0, \pi)$ and with an integration by parts, we obtain for any $g_{1}, g_{2} \in L_{0}^{\infty}(0, \pi)$ the equality:

$$
\begin{aligned}
0=\int_{\Omega_{2}} u_{1}\left(x_{1}, x_{2}\right)\left(\int_{0}^{x_{1}} g_{1}(s) d s\right) & g_{2}\left(x_{2}\right) d x-\int_{\Omega_{2}} u_{2}\left(x_{1}, x_{2}\right) g_{1}\left(x_{1}\right)\left(\int_{0}^{x_{2}} g_{2}(s) d s\right) d x \\
& =\int_{\Omega_{2}}\left(-\int_{0}^{x_{1}} u_{1}\left(s, x_{2}\right) d s+\int_{0}^{x_{2}} u_{2}\left(x_{1}, s\right) d s\right) g_{1}\left(x_{1}\right) g_{2}\left(x_{2}\right) d x
\end{aligned}
$$

Thus, using Lemma 2.3 for $p=2$ and $\Theta=\Omega_{2}$, we have that there are $h_{1}, h_{2} \in L^{2}(0, \pi)$ such that:

$$
-\int_{0}^{x_{1}} u_{1}\left(s, x_{2}\right) d s+\int_{0}^{x_{2}} u_{2}\left(x_{1}, s\right) d s=h_{1}\left(x_{1}\right)+h_{2}\left(x_{2}\right) .
$$

If we differentiate with respect to $\partial_{x_{1} x_{2}}$, we have that:

$$
0=-\partial_{x_{2}} u_{1}\left(x_{1}, x_{2}\right)+\partial_{x_{1}} u_{2}\left(x_{1}, x_{2}\right)=\nabla \times u \text {. }
$$

In addition, since $u \in \mathcal{H}\left(\Omega_{2}\right)$, we also have that $\nabla \cdot u=0$ and $u \cdot n=0$. Consequently, since $\Omega_{2}$ is Lipschitz and simply connected, we have that $u=0$ (see, for instance, [1, Lemma IV.4.6]).

\subsection{A total set of $\mathcal{H}\left(\Omega_{2}\right)$ formed by solutions of 1.5}

Throughout this section we focus on getting all the solutions of 1.5 (in $\Omega_{2}$ ). In particular, we first get the candidates and then prove that they form a total set:

Proposition 2.5. For all $m=\left(m_{1}, m_{2}\right) \in\left(\mathbb{N}_{*}\right)^{2}$ let us set:

$$
u_{m}(x):=\left(\int_{0}^{x_{1}} g_{1, m_{1}}(s) d s g_{2, m_{2}}\left(x_{2}\right),-g_{1, m_{1}}\left(x_{1}\right) \int_{0}^{x_{2}} g_{2, m_{2}}(s) d s\right)
$$

for

$$
g_{1, m_{1}}(s):=\cos \left(m_{1} s\right), \quad g_{2, m_{2}}(s):=\left(2 m_{2} \varepsilon \cos \left(m_{2} s\right)-\sin \left(m_{2} s\right)\right) e^{-(2 \varepsilon)^{-1} s} .
$$


Then, $u_{m}$ is a solution of the system (1.5) (in $\Omega_{2}$ ) such that:

$$
\left\|u_{m}\right\|_{\mathbf{L}^{2}\left(\Omega_{2}\right)}=\sqrt{\frac{\left(1-e^{-\pi \varepsilon^{-1}}\right) m_{2}^{2} \pi \varepsilon^{3}\left(1+4 \varepsilon^{2}\left(m_{1}^{2}+m_{2}^{2}\right)\right)}{m_{1}^{2}+4 m_{1}^{2} m_{2}^{2} \varepsilon^{2}}},
$$

whose associated pressure is constant and whose associated eigenvalue is:

$$
\lambda_{m}=\left(m_{1}^{2}+m_{2}^{2}\right) \varepsilon+\frac{1}{4 \varepsilon} .
$$

Remark 2.6. We have the equality:

$$
u_{m}(x)=e^{-(2 \varepsilon)^{-1} x_{2}}\left(\frac{\sin \left(m_{1} x_{1}\right)}{m_{1}}\left(2 m_{2} \varepsilon \cos \left(m_{2} x_{2}\right)-\sin \left(m_{2} x_{2}\right)\right),-2 \varepsilon \cos \left(m_{1} x_{1}\right) \sin \left(m_{2} x_{2}\right)\right) .
$$

Moreover, using that $m_{1}, m_{2} \geq 1$ and $\varepsilon \in(0,1)$, we obtain from 2.18$)$ the estimate:

$$
\left\|u_{m}\right\|_{\mathbf{L}^{\infty}\left(\Omega_{2}\right)} \leq C\left(m_{2} \varepsilon+1\right) .
$$

Proof. We can check directly the conclusions of Proposition 2.5 for the functions given in (2.18). In addition, we can get all the eigenfunctions of the elliptic system associated to (1.7) and (1.6) and find the pairs of compatibility. However, we show an "intuitive" way to get the $u_{m}$ to provide a better insight to the reader which can help him/her to understand what happens in other Stokes systems. With that purpose, we look for eigenfunctions in $S V\left(\Omega_{2}\right)$ (see 2.1$)$ ) such that $g_{1}, g_{2} \in C^{\infty}([0, \pi])$. We can prove easily that in that case the condition $[1.5]_{3}$ is equivalent to (if $g_{1} \neq 0$ and $g_{2} \neq 0$ ):

$$
g_{1}^{\prime}(0)=g_{1}^{\prime}(\pi)=\varepsilon g_{2}^{\prime}(0)+g_{2}(0)=\varepsilon g_{2}^{\prime}(\pi)+g_{2}(\pi)=0 .
$$

If we apply the divergence operator to $(1.5)_{1}$, we obtain that $\Delta p=0$. In particular, we can apply the Laplacian operator to $(1.5)_{1}$ to get possible solutions. Indeed, since:

$$
\begin{aligned}
\Delta u_{1} & =g_{1}^{\prime}\left(x_{1}\right) g_{2}\left(x_{2}\right)+\int_{0}^{x_{1}} g_{1}(s) d s g_{2}^{\prime \prime}\left(x_{2}\right), \\
\partial_{x_{2}} \Delta u_{1} & =g_{1}^{\prime}\left(x_{1}\right) g_{2}^{\prime}\left(x_{2}\right)+\int_{0}^{x_{1}} g_{1}(s) d s g_{2}^{\prime \prime \prime}\left(x_{2}\right), \\
\Delta^{2} u_{1} & =g_{1}^{\prime \prime \prime}\left(x_{1}\right) g_{2}\left(x_{2}\right)+2 g_{1}^{\prime}\left(x_{1}\right) g_{2}^{\prime \prime}\left(x_{2}\right)+\int_{0}^{x_{1}} g_{1}(s) d s g_{2}^{i v)}\left(x_{2}\right) ;
\end{aligned}
$$

we have that:

$$
\begin{aligned}
-\varepsilon g_{1}^{\prime \prime \prime}\left(x_{1}\right) g_{2}\left(x_{2}\right)-2 \varepsilon g_{1}^{\prime}\left(x_{1}\right) g_{2}^{\prime \prime}\left(x_{2}\right) & -\varepsilon \int_{0}^{x_{1}} g_{1}(s) d s g_{2}^{i v)}\left(x_{2}\right)-g_{1}^{\prime}\left(x_{1}\right) g_{2}^{\prime}\left(x_{2}\right) \\
& -\int_{0}^{x_{1}} g_{1}(s) d s g_{2}^{\prime \prime \prime}\left(x_{2}\right)-\lambda g_{1}^{\prime}\left(x_{1}\right) g_{2}\left(x_{2}\right)-\lambda \int_{0}^{x_{1}} g_{1}(s) d s g_{2}^{\prime \prime}\left(x_{2}\right)=0
\end{aligned}
$$

We have that $g_{1, m_{1}} \in L_{0}^{2}(0, \pi)$ (see 2.15$)$ ), that $g_{1, m_{1}}$ satisfies the first two conditions of 2.20 , and that $g_{1, m_{1}}$ is an eigenfunction of the Laplacian; so it is a reasonable function to do an attempt. Under that choice, the functions $g_{2} \in L_{0}^{2}(0, \pi)$ have to satisfy the two last conditions of 2.20) and the ODE:

$$
-\varepsilon m_{1}^{4} g_{2}+2 \varepsilon m_{1}^{2} g_{2}^{\prime \prime}-\varepsilon g_{2}^{i v)}+m_{1}^{2} g_{2}^{\prime}-g_{2}^{\prime \prime \prime}+\lambda m_{1}^{2} g_{2}-\lambda g_{2}^{\prime \prime}=0 .
$$

In order to solve 2.21) we calculate the roots of its characteristic polynomial:

$$
-\varepsilon\left(r^{2}-m_{1}^{2}\right)^{2}-r\left(r^{2}-m_{1}^{2}\right)-\lambda\left(r^{2}-m_{1}^{2}\right)=0 .
$$


Let us focus on the roots of:

$$
r^{2}+\frac{r}{\varepsilon}+\frac{\lambda}{\varepsilon}-m_{1}^{2}=0
$$

which are given by:

$$
r=\frac{-\frac{1}{\varepsilon} \pm \sqrt{\frac{1}{\varepsilon^{2}}-4 \frac{\lambda}{\varepsilon}+4 m_{1}^{2}}}{2} .
$$

These roots are complex if $\lambda$ is sufficiently large with respect to $\varepsilon^{-1}$. We then have that the associated solutions for $\lambda$ large enough are:

$$
g_{2, m_{2}}(s):=\left(A \cos \left(m_{2} s\right)+B \sin \left(m_{2} s\right)\right) e^{-(2 \varepsilon)^{-1} s},
$$

where $m_{2}>0$ is defined by:

$$
m_{2}:=m_{2}\left(\varepsilon, \lambda, m_{1}\right):=\sqrt{\frac{\lambda}{\varepsilon}-\frac{1}{4 \varepsilon^{2}}-m_{1}^{2}} .
$$

Consequently, the $g_{2, m_{2}}$ that we get are solutions of:

$$
-\varepsilon g_{2}^{\prime \prime}-g_{2}^{\prime}=\left(\lambda-\varepsilon m_{1}^{2}\right) g_{2}
$$

We are going to see that there are some $g_{2, m_{2}} \in L_{0}^{2}(0, \pi)$ that also satisfy the last two conditions of (2.20. Indeed, we have that:

$$
g_{2, m_{2}}^{\prime}(s)=\left[\left(-\frac{A}{2 \varepsilon}+m_{2} B\right) \cos \left(m_{2} s\right)+\left(-\frac{B}{2 \varepsilon}-A m_{2}\right) \sin \left(m_{2} s\right)\right] e^{-(2 \varepsilon)^{-1} s} .
$$

From $g_{2, m_{2}}(0)=-\varepsilon g_{2, m_{2}}^{\prime}(0)$, we obtain that:

$$
A=-2 m_{2} \varepsilon B .
$$

Moreover, from $g_{2, m_{2}}(\pi)=-\varepsilon g_{2, m_{2}}^{\prime}(\pi)$ and 2.27 we have that:

$$
-2 m_{2} \varepsilon B \cos \left(m_{2} \pi\right)+B \sin \left(m_{2} \pi\right)=-2 m_{2} \varepsilon B \cos \left(m_{2} \pi\right)+\left(\frac{1}{2}-2 m_{2}^{2} \varepsilon^{2}\right) B \sin \left(m_{2} \pi\right) .
$$

Consequently,

$$
B \sin \left(m_{2} \pi\right)=-4 m_{2}^{2} \varepsilon^{2} B \sin \left(m_{2} \pi\right) .
$$

Hence, we have either $m_{2} \in \mathbb{N}_{\star}$ or $B=0$, which by (2.27) implies that $g_{2, m_{2}}=0$, which just gives the null solution. Consequently, the possible values for $g_{2, m_{2}}$ are (we have taken $B=-1$ ):

$$
g_{2, m_{2}}(s)=\left(2 m_{2} \varepsilon \cos \left(m_{2} s\right)-\sin \left(m_{2} s\right)\right) e^{-(2 \varepsilon)^{-1} s}=\frac{d\left(2 \varepsilon \sin \left(m_{2} s\right) e^{-(2 \varepsilon)^{-1} s}\right)}{d s}, \quad \forall m_{2} \in \mathbb{N}_{*},
$$

which clearly belong to $L_{0}^{2}(0, \pi)$. Thus, our candidates are those defined in 2.15 and $\lambda_{m}$ satisfies 2.17) because of 2.25 .

In order to see that $u_{m}$ satisfies the first equation of $(1.5)_{1}$ with a constant pressure for any given time, we have to use 2.14), that $g_{2, m_{2}}$ is a solution of 2.26 and that $g_{1, m_{1}}$ is a solution of $-\varepsilon g_{1, m_{1}}^{\prime \prime}=\varepsilon m_{1}^{2} g_{1, m_{1}}$. Moreover, in order to see that they satisfy the second equation of $(1.5)_{1}$, we consider 2.26) and that $\varepsilon g_{2, m_{2}}^{\prime}(0)+g_{2, m_{2}}(0)=0$, which imply that:

$$
\left(\lambda-\varepsilon m_{1}^{2}\right) \int_{0}^{s} g_{2, m_{2}}\left(s^{\prime}\right) d s^{\prime}=-\varepsilon g_{2, m_{2}}^{\prime}-g_{2, m_{2}}=-\varepsilon \frac{d^{2}\left(\int_{0}^{s} g_{2, m_{2}}\left(s^{\prime}\right) d s^{\prime}\right)}{d s^{2}}-\frac{d\left(\int_{0}^{s} g_{2, m_{2}}\left(s^{\prime}\right) d s^{\prime}\right)}{d s} .
$$


Finally, let us prove (2.16) using (2.18). We recall that, if $a, b \in \mathbb{R}$ such that $a \neq 0$ or $b \neq 0$ :

$$
\int_{0}^{s} \sin \left(a s^{\prime}\right) e^{b s^{\prime}} d s^{\prime}=\frac{e^{b s}(b \sin (a s)-a \cos (a s))+a}{a^{2}+b^{2}}, \quad \int_{0}^{s} \cos \left(a s^{\prime}\right) e^{b s^{\prime}} d s^{\prime}=\frac{e^{b s}(b \cos (a s)+a \sin (a s))-b}{a^{2}+b^{2}} \text {. }
$$

In addition, recalling that $m_{1} \in \mathbb{N}_{*}$, we have the identity:

$$
\int_{0}^{\pi} \sin ^{2}\left(m_{1} x_{1}\right) d x_{1}=\frac{\pi}{2} .
$$

Similarly, we have that:

$$
\begin{aligned}
\left(2 m_{2} \varepsilon \cos \left(m_{2} x_{2}\right)-\sin \left(m_{2} x_{2}\right)\right)^{2}=2 m_{2}^{2} \varepsilon^{2}\left(1+\cos \left(2 m_{2} x_{2}\right)\right)-2 m_{2} \varepsilon \sin \left(2 m_{2} x_{2}\right)+\frac{\left(1-\cos \left(2 m_{2} x_{2}\right)\right)}{2} \\
=\frac{4 m_{2}^{2} \varepsilon^{2}+1}{2}+\frac{4 m_{2}^{2} \varepsilon^{2}-1}{2} \cos \left(2 m_{2} x_{2}\right)-2 m_{2} \varepsilon \sin \left(2 m_{2} x_{2}\right) .
\end{aligned}
$$

Consequently, considering that $m_{2} \in \mathbb{N}_{*}, 2.29$ and 2.31 we obtain the equality:

$$
\begin{aligned}
\int_{0}^{\pi}\left(2 m_{2} \varepsilon \cos \right. & \left.\left(m_{2} x_{2}\right)-\sin \left(m_{2} x_{2}\right)\right)^{2} e^{-\varepsilon^{-1} x_{2}} d x_{2} \\
= & \int_{0}^{\pi}\left(\frac{4 m_{2}^{2} \varepsilon^{2}+1}{2}+\frac{4 m_{2}^{2} \varepsilon^{2}-1}{2} \cos \left(2 m_{2} x_{2}\right)-2 m_{2} \varepsilon \sin \left(2 m_{2} x_{2}\right)\right) e^{-\varepsilon^{-1} x_{2}} d x_{2} \\
= & \varepsilon \frac{4 m_{2}^{2} \varepsilon^{2}+1}{2}\left(1-e^{-\pi \varepsilon^{-1}}\right)+\frac{4 m_{2}^{2} \varepsilon^{2}-1}{2} \frac{\varepsilon^{-1}\left(1-e^{-\pi \varepsilon^{-1}}\right)}{4 m_{2}^{2}+\varepsilon^{-2}}-2 m_{2} \varepsilon \frac{2 m_{2}\left(1-e^{-\pi \varepsilon^{-1}}\right)}{4 m_{2}^{2}+\varepsilon^{-2}} \\
& =\frac{\left(4 m_{2}^{2} \varepsilon^{3}+\varepsilon\right)\left(4 m_{2}^{2} \varepsilon^{2}+1\right)+4 m_{2}^{2} \varepsilon^{3}-\varepsilon-8 m_{2}^{2} \varepsilon^{3}}{8 m^{2} \varepsilon^{2}+2}\left(1-e^{-\pi \varepsilon^{-1}}\right)=2 m_{2}^{2} \varepsilon^{3}\left(1-e^{-\pi \varepsilon^{-1}}\right) .
\end{aligned}
$$

Moreover, since $m_{1} \in \mathbb{N}$ we have the identity:

$$
\int_{0}^{\pi} \cos ^{2}\left(m_{1} x_{1}\right) d x_{1}=\frac{\pi}{2} .
$$

In addition, from $m_{2} \in \mathbb{N}_{*}$ we obtain the equality:

$$
\begin{array}{r}
\int_{0}^{\pi} \sin ^{2}\left(m_{2} x_{2}\right) e^{-\varepsilon^{-1} x_{2}} d x_{2}=\int_{0}^{\pi} \frac{1-\cos \left(2 m_{2} x_{2}\right)}{2} e^{-\varepsilon^{-1} x_{2}}=\frac{1}{2}\left(\varepsilon-\frac{\varepsilon}{4 m_{2}^{2} \varepsilon^{2}+1}\right)\left(1-e^{-\pi \varepsilon^{-1}}\right) \\
=\frac{2 m_{2}^{2} \varepsilon^{3}}{4 m_{2}^{2} \varepsilon^{2}+1}\left(1-e^{-\pi \varepsilon^{-1}}\right) .
\end{array}
$$

So, combining 2.18), 2.30, 2.32, 2.33) and 2.34 we obtain that:

$$
\begin{aligned}
\left\|u_{m}\right\|_{\mathbf{L}^{2}(\Omega)}^{2}=\frac{\pi}{m_{1}^{2}} & m_{2}^{2} \varepsilon^{3}\left(1-e^{-\pi \varepsilon^{-1}}\right)+\frac{4 \pi m_{2}^{2} \varepsilon^{5}}{4 m_{2}^{2} \varepsilon^{2}+1}\left(1-e^{-\pi \varepsilon^{-1}}\right) \\
& =\left(1-e^{-\pi \varepsilon^{-1}}\right) \pi \frac{4 m_{2}^{4} \varepsilon^{5}+m_{2}^{2} \varepsilon^{3}+4 m_{2}^{2} m_{1}^{2} \varepsilon^{5}}{4 m_{2}^{2} m_{1}^{2} \varepsilon^{2}+m_{1}^{2}}=\frac{\left(1-e^{-\pi \varepsilon^{-1}}\right) m_{2}^{2} \pi \varepsilon^{3}\left(1+4 \varepsilon^{2}\left(m_{1}^{2}+m_{2}^{2}\right)\right)}{m_{1}^{2}+4 m_{1}^{2} m_{2}^{2} \varepsilon^{2}},
\end{aligned}
$$

which implies 2.16).

Proposition 2.7. The set $\left\{u_{m}\right\}_{m \in\left(\mathbb{N}_{*}\right)^{2}}$ is a total set.

Proof. Thanks to Proposition 2.1, it suffices to prove that:

$$
S V\left(\Omega_{2}\right) \subset \overline{\operatorname{span}\left\{u_{m}\right\}} .
$$

We prove this inclusion constructively; that is, writing an element of $S V\left(\Omega_{2}\right)$ as a limit of linear combinations of the eigenfunctions $u_{m}$. We consider a function $u \in S V\left(\Omega_{2}\right)$ (see (2.1)). 
Let us recall that $\left\{\frac{\cos (k s)}{\sqrt{2^{-1} \pi}}\right\}_{k \in \mathbb{N}_{*}} \cup\left\{\pi^{-1}\right\}$ is an orthonormal basis of $L^{2}(0, \pi)$ (the basis obtained by the diagonalization of the Laplacian with Neumann boundary conditions in $(0, \pi))$. So, since $g_{1} \in L_{0}^{2}(0, \pi)$, we have that, for some $a_{k} \in l^{2}$ :

$$
g_{1}(s)=\sum_{k \geq 1} a_{k} \frac{\cos (k s)}{\sqrt{2^{-1} \pi}} .
$$

That series must be understood as a limit in $L^{2}(0, \pi)$.

Next, we remark that:

$$
\left\{\frac{\gamma_{k}^{\varepsilon}}{\sqrt{2^{-1} \pi} \sqrt{1+4 k^{2} \varepsilon^{2}}}\right\}_{k \geq 1} \cup\left\{\frac{e^{-(2 \varepsilon)^{-1} s}}{\sqrt{\varepsilon\left(1-e^{-\pi / \varepsilon}\right)}}\right\}
$$

for:

$$
\gamma_{k}^{\varepsilon}(s):=2 k \varepsilon \cos (k s)-\sin (k s),
$$

is the orthonormal basis obtained by diagonalization of the Laplacian in $(0, \pi)$ with Robin boundary conditions:

$$
\left\{\begin{array}{l}
-2 \varepsilon g^{\prime}(0)=g(0), \\
-2 \varepsilon g^{\prime}(\pi)=g(\pi) .
\end{array}\right.
$$

Indeed, we have that the Laplacian is self-adjoint in the subspace of $H^{2}\left(\Omega_{2}\right)$ that satisfies 2.39 . Thus, we have, for some $b_{k} \in l^{2}$ :

$$
g_{2}(s)=\left(g_{2}(s) e^{(2 \varepsilon)^{-1} s}\right) e^{-(2 \varepsilon)^{-1} s}=\sum_{k \geq 1} b_{k} \frac{\gamma_{k}^{\varepsilon}(s) e^{-(2 \varepsilon)^{-1} s}}{\sqrt{2^{-1} \pi} \sqrt{1+4 k^{2} \varepsilon^{2}}} .
$$

We have used that $g_{2} \in L_{0}^{2}(0, \pi)$ implies that $g_{2} e^{(2 \varepsilon)^{-1} s}$ is orthogonal to $e^{-(2 \varepsilon)^{-1} s}$. Moreover, we have to understand the series as a limit in $L^{2}(0, \pi)$, not as a pointwise one. In that sense, we can place the exponential into the series because the multiplication of the functions of $L^{2}(0, \pi)$ by an element of $L^{\infty}(0, \pi)$ is a continuous linear form.

Next, we recall that the operator $\int_{0}^{s}$ is a continuous endomorphism in $L^{2}(0, \pi)$. Thus, we have that:

$$
\int_{0}^{s} g_{1}\left(s^{\prime}\right) d s^{\prime}=\sum_{k \geq 1} a_{k} \frac{\sin (k s)}{k \sqrt{2^{-1} \pi}} .
$$

Similarly, we have that:

$$
\int_{0}^{s} g_{2}\left(s^{\prime}\right) d s^{\prime}=\sum_{k \geq 1} b_{k} \frac{2 \varepsilon \sin (k s) e^{-(2 \varepsilon)^{-1} s}}{\sqrt{2^{-1} \pi} \sqrt{1+4 k^{2} \varepsilon^{2}}} .
$$

The limits in 2.41 and 2.42 are both pointwise and in $L^{2}(0, \pi)$.

We finally recall that the tensor product of two convergent sequences of $L^{2}(0, \pi)$ converges in $L^{2}\left(\Omega_{2}\right)$ to the tensor product of their respective limits. Thus, we have that 2.36, $2.40,2.41$ and 2.42 imply:

$$
\int_{0}^{x_{1}} g_{1}(s) d s g_{2}\left(x_{2}\right)=\lim _{k \rightarrow \infty} \sum_{m_{1}, m_{2} \geq 1}^{k} \frac{2 a_{m_{1}} b_{m_{2}}}{\pi \sqrt{1+4 m_{2}^{2} \varepsilon^{2}}} \frac{\sin \left(m_{1} x_{1}\right)}{m_{1}} \gamma_{m_{2}}^{\varepsilon}\left(x_{2}\right) e^{-(2 \varepsilon)^{-1} x_{2}},
$$


and that:

$$
-g_{1}\left(x_{1}\right) \int_{0}^{x_{2}} g_{2}(s) d s=\lim _{k \rightarrow \infty} \sum_{m_{1}, m_{2} \geq 1}^{k} \frac{2 a_{m_{1}} b_{m_{2}}}{\pi \sqrt{1+4 m_{2}^{2} \varepsilon^{2}}}\left(-2 \varepsilon \cos \left(m_{1} x_{1}\right) \sin \left(m_{2} x_{2}\right) e^{-(2 \varepsilon)^{-1} x_{2}}\right) .
$$

Consequently, we have that:

$$
u=\lim _{k \rightarrow \infty} \sum_{m_{1}, m_{2} \geq 1}^{k} \frac{2 a_{m_{1}} b_{m_{2}}}{\pi \sqrt{1+4 m_{2}^{2} \varepsilon^{2}}} u_{m}
$$

which proves 2.35).

\subsection{Properties of the solution of 1.3 in $\Omega_{2}$}

Since our operator is not self-adjoint, the total set $\left\{u_{m}\right\}$ given in 2.18 does not have to be orthogonal (and it is not). In addition, it is not immediate to write an element of $\mathcal{H}\left(\Omega_{2}\right)$ as a series of elements proportional to those in $\left\{u_{m}\right\}$. Indeed, there are total sets in $l^{2}$ (for instance $\left\{e_{1}\right\} \cup\left\{e_{1}+e_{k}\right\}_{k \geq 2}$ ) such that it is not possible to write any element of $l^{2}$ (for instance $\left(k^{-1}\right)_{k \geq 1}$ ) as a series of elements proportional to those in the total sets. In our particular situation we do not even know if this is possible (probably yes), but we will prove that at least we can express the solutions of 1.3 in $\Omega_{2}$ with the help of $\left\{u_{m}\right\}$.

The next step is given any $u \in \operatorname{span}\left\{u_{m}\right\}$, to get its coordinates in our generating system. In order to do so, we get a set of functions $v_{m} \in \mathbf{L}^{2}\left(\Omega_{2}\right)$ such that $\left\{\left(u_{m}, v_{m}\right)\right\}$ is a bi-orthogonal system; that is, such that:

$$
\int_{\Omega_{2}} u_{m}(x) \cdot v_{m^{\prime}}(x) d x=1_{m=m^{\prime}} .
$$

Usually, we can get those functions $v_{m}$ by diagonalization of the adjoint operator, but it is easier to do a systematic search in $\mathbf{L}^{2}\left(\Omega_{2}\right)$. In that sense we define (recall 2.38 ):

$$
v_{m}(x):=\frac{2}{\pi^{2}} e^{(2 \varepsilon)^{-1} x_{2}}\left(m_{1} \sin \left(m_{1} x_{1}\right) \frac{\gamma_{m_{2}}^{\varepsilon}\left(x_{2}\right)}{1+4 m_{2}^{2} \varepsilon^{2}},-\frac{\cos \left(m_{1} x_{1}\right) \sin \left(m_{2} x_{2}\right)}{2 \varepsilon}\right) .
$$

We can easily verify 2.43 because $\left\{\sqrt{2 \pi^{-1}} \sin (k s)\right\}_{k \in \mathbb{N}_{*}},\left\{\sqrt{2 \pi^{-1}} \cos (k s)\right\}_{k \in \mathbb{N}_{*}} \cup\left\{\pi^{-1}\right\}$ and 2.37 are Hilbert basis in $L^{2}(0, \pi)$. We remark that, for a constant $C$ that does not depend on $m_{1}, m_{2}$ or $\varepsilon$ :

$$
\left\|v_{m}\right\|_{\mathbf{L}^{2}\left(\Omega_{2}\right)} \leq C\left(m_{1}+\frac{1}{\varepsilon}\right) e^{(2 \varepsilon)^{-1} \pi} .
$$

Now we are ready to get the solutions of 1.3 through a spectral decomposition.

Proposition 2.8. Let $\varphi^{T} \in \mathcal{H}\left(\Omega_{2}\right)$. Let us denote, for $t \leq T, L_{\varepsilon}^{T}(t) \varphi^{T}$ the value in $\mathcal{H}\left(\Omega_{2}\right)$ of the energy solution of system (1.3) in $\Omega_{2}$ on time $t$. Then, we have that:

1. For all $t<T$ and $\varepsilon>0$ :

$$
L_{\varepsilon}^{T}(t) \varphi^{T}=\sum_{m \in\left(\mathbb{N}_{*}\right)^{2}}\left\langle\varphi^{T}, v_{m}\right\rangle_{\mathbf{L}^{2}\left(\Omega_{2}\right)} \exp \left[\left(\left(m_{1}^{2}+m_{2}^{2}\right) \varepsilon+\frac{1}{4 \varepsilon}\right)(t-T)\right] u_{m} .
$$


In particular, the series of the right hand-side of (2.46) is well-defined and absolutely convergent in $\mathcal{H}\left(\Omega_{2}\right)$.

2. For all $\delta>0$ there is $C_{\delta}>0$ such that for all $T>0, \varphi^{T} \in \mathcal{H}\left(\Omega_{2}\right)$, $\varepsilon \in(0,1)$ and $s \leq T-2 \pi-\delta$ :

$$
\left\|L_{\varepsilon}^{T}(s) \varphi^{T}\right\|_{\mathbf{L}^{2}\left(\Omega_{2}\right)} \leq C_{\delta} \exp \left[\frac{s-(T-2 \pi-\delta)}{4 \varepsilon}\right]\left\|\varphi^{T}\right\|_{\mathbf{L}^{2}\left(\Omega_{2}\right)} .
$$

Throughout the proof we denote $C_{\varepsilon, t}$ a generic constant which might be different each time and which just depends on $\varepsilon$ and $t$.

Proof. First of all, we have that 2.46 is true if $\varphi^{T} \in \operatorname{span}\left\{u_{m}\right\}$ as a consequence of Proposition 2.5 (see 2.17) and 2.43. Moreover, for $t>0$ and $\varepsilon>0$ fixed, $L_{\varepsilon}^{T}(t)$ is a continuous endomorphism on $\mathcal{H}\left(\Omega_{2}\right)$. Consequently in order to prove Item 1, it suffices to prove that the series of the right-hand side of (2.46) is well-defined, absolutely convergent and continuous. We shall prove the three properties at once.

Recalling estimate 2.19 , estimate 2.45 and that $x e^{-x^{2}}$ is bounded in $\mathbb{R}^{+}$, we have that, if $\varepsilon \in(0,1)$ :

$$
\begin{aligned}
& \sum_{m \in\left(\mathbb{N}_{*}\right)^{2}}\left|\left\langle\varphi^{T}, v_{m}\right\rangle_{\mathbf{L}^{2}\left(\Omega_{2}\right)}\right| \exp \left[\left(\left(m_{1}^{2}+m_{2}^{2}\right) \varepsilon+\frac{1}{4 \varepsilon}\right)(t-T)\right]\left\|u_{m}\right\|_{\mathbf{L}^{2}\left(\Omega_{2}\right)} \\
& \leq C_{\varepsilon, t}\left\|\varphi^{T}\right\|_{\mathbf{L}^{2}\left(\Omega_{2}\right)} \sum_{m \in\left(\mathbb{N}_{*}\right)^{2}} m_{1} m_{2} \exp \left(\left(m_{1}^{2}+m_{2}^{2}\right) \varepsilon(t-T)\right) \\
& \quad \leq C_{\varepsilon, t}\left\|\varphi^{T}\right\|_{\mathbf{L}^{2}\left(\Omega_{2}\right)} \sum_{m \in\left(\mathbb{N}_{*}\right)^{2}} \exp \left(\left(m_{1}^{2}+m_{2}^{2}\right) \frac{\varepsilon(t-T)}{2}\right) .
\end{aligned}
$$

The series in the right-hand side of 2.48 is convergent because, in $\left(\mathbb{R}^{+}\right)^{2}$ :

$$
\begin{aligned}
\exp \left(\left(m_{1}^{2}+m_{2}^{2}\right) \frac{\varepsilon(t-T)}{2}\right) 1_{\left[m_{1}-1, m_{1}\right] \times\left[m_{2}-1, m_{2}\right]}\left(x_{1}, x_{2}\right) & \\
& \leq \exp \left(\left(x_{1}^{2}+x_{2}^{2}\right) \frac{\varepsilon(t-T)}{2}\right) 1_{\left[m_{1}-1, m_{1}\right] \times\left[m_{2}-1, m_{2}\right]}\left(x_{1}, x_{2}\right)
\end{aligned}
$$

which implies that:

$$
\sum_{m \in\left(\mathbb{N}_{*}\right)^{2}} \exp \left(\left(m_{1}^{2}+m_{2}^{2}\right) \frac{\varepsilon(t-T)}{2}\right) \leq \int_{\left(\mathbb{R}^{+}\right)^{2}} \exp \left(\left(x_{1}^{2}+x_{2}^{2}\right) \frac{\varepsilon(t-T)}{2}\right) d x=\frac{2}{\varepsilon(T-t)} \frac{\pi}{4} .
$$

Combining 2.48) and 2.49, we have that:

$$
\sum_{m \in\left(\mathbb{N}_{*}\right)^{2}}\left|\left\langle\varphi^{T}, v_{m}\right\rangle_{\mathbf{L}^{2}\left(\Omega_{2}\right)}\right| \exp \left[\left(\left(m_{1}^{2}+m_{2}^{2}\right) \varepsilon+\frac{1}{4 \varepsilon}\right)(t-T)\right]\left\|u_{m}\right\|_{\mathbf{L}^{2}\left(\Omega_{2}\right)} \leq C_{\varepsilon, t}\left\|\varphi^{T}\right\|_{\mathbf{L}^{2}\left(\Omega_{2}\right)},
$$

which implies that the series in the right-hand side of 2.46 is absolutely convergent and thus well-defined. Moreover, since the right-hand side of $(2.46)$ is linear, 2.50$)$ implies that the right-hand side of (2.46) is continuous in $\mathcal{H}\left(\Omega_{2}\right)$. 
Finally, let us prove 2.47). Indeed, using (2.19), the triangular inequality, and Cauchy-Schwarz, we have that, for all $\varepsilon \in(0,1)$ :

$$
\left\|L_{\varepsilon}^{T}(s) \varphi^{T}\right\|_{\mathbf{L}^{2}\left(\Omega_{2}\right)} \leq C\left\|\varphi^{T}\right\|_{\mathbf{L}^{2}\left(\Omega_{2}\right)} \sum_{m \in\left(\mathbb{N}_{*}\right)^{2}} m_{2}\left\|v_{m}\right\|_{\mathbf{L}^{2}\left(\Omega_{2}\right)} \exp \left[\left(\left(m_{1}^{2}+m_{2}^{2}\right) \varepsilon+\frac{1}{4 \varepsilon}\right)(s-T)\right] .
$$

Next, using 2.45 and that $x e^{-x^{2}}$ is bounded in $\mathbb{R}^{+}$, if $s \leq T-2$ and if $\varepsilon \in(0,1)$, we have that 2.51) turns into:

$$
\left\|L_{\varepsilon}^{T}(s) \varphi^{T}\right\|_{\mathbf{L}^{2}\left(\Omega_{2}\right)} \leq \frac{C\left\|\varphi^{T}\right\|_{\mathbf{L}^{2}\left(\Omega_{2}\right)}}{\varepsilon^{2}}\left[\sum_{m \in\left(\mathbb{N}_{*}\right)^{2}} \exp \left(-\left(m_{1}^{2}+m_{2}^{2}\right) \varepsilon\right)\right] \exp \left(\frac{s-(T-2 \pi)}{4 \varepsilon}\right) .
$$

Using 2.49 for $t=T-2$, we have that there is $C_{\delta}>0$ such that for all $\varepsilon \in(0,1)$ :

$$
\frac{1}{\varepsilon^{2}} \sum_{m \in\left(\mathbb{N}_{*}\right)^{2}} \exp \left(-\left(m_{1}^{2}+m_{2}^{2}\right) \varepsilon\right) \leq \frac{\pi}{4 \varepsilon^{3}} \leq C_{\delta} e^{\delta(4 \varepsilon)^{-1}} .
$$

Consequently, 2.52 implies 2.47.

Remark 2.9. Let $T>0$ and $\varphi^{T} \in \mathcal{H}\left(\Omega_{2}\right)$. Then, the application:

$$
\mathbb{R}_{*}^{+} \ni \varepsilon \mapsto \varphi^{\varepsilon}(0, \cdot) \in \mathcal{H}\left(\Omega_{2}\right)
$$

(for $\varphi^{\varepsilon}$ the solution of 1.3$)$ in $\Omega_{2}$ and initial value $\varphi^{T}$ ) is continuous. To prove the continuity, we fix $\varepsilon_{0}>0$ and $\varrho>0$ and verify that if $\delta>0$ is small enough, we have for all $\varepsilon \in\left(\varepsilon_{0}-\delta, \varepsilon_{0}+\delta\right)$ the following inequality:

$$
\left\|\varphi^{\varepsilon}(0, \cdot)-\varphi^{\varepsilon_{0}}(0, \cdot)\right\|_{\mathbf{L}^{2}\left(\Omega_{2}\right)} \leq \varrho .
$$

First, using 2.19), 2.45) and that $s e^{-s^{2}} \in L^{\infty}\left(\mathbb{R}^{+}, d s\right)$ we obtain that for all $\varepsilon \in\left(\varepsilon_{0} / 2,3 \varepsilon_{0} / 2\right)$ and $M \in \mathbb{N}$ :

$$
\begin{aligned}
\left\|\sum_{m_{1}+m_{2} \geq M}\left\langle\varphi^{T}, v_{m}^{\varepsilon}\right\rangle_{\mathbf{L}^{2}\left(\Omega_{2}\right)} \exp \left[-\left(\left(m_{1}^{2}+m_{2}^{2}\right) \varepsilon+\frac{1}{4 \varepsilon}\right) T\right] u_{m}^{\varepsilon}\right\|_{\mathbf{L}^{2}\left(\Omega_{2}\right)} \\
\leq C\left(T, \varphi^{T}\right)\left(1+\frac{1}{\varepsilon^{2}}\right) \exp \left(\frac{-T+2 \pi}{4 \varepsilon}\right)\left[\sum_{m_{1}+m_{2} \geq M} \exp \left(-\left(m_{1}^{2}+m_{2}^{2}\right) \frac{\varepsilon T}{2}\right)\right] \\
\leq C\left(T, \varphi^{T}, \varepsilon_{0}\right)\left[\sum_{m_{1}+m_{2} \geq M} \exp \left(-\left(m_{1}^{2}+m_{2}^{2}\right) \frac{\varepsilon_{0} T}{4}\right)\right] .
\end{aligned}
$$

Consequently, since the series in the right-hand of 2.54 is convergent, there is $M\left(T, \varphi^{T}, \varepsilon_{0}\right)$ large enough such that for all $\varepsilon \in\left(\varepsilon_{0} / 2,3 \varepsilon_{0} / 2\right)$ :

$$
\left\|\sum_{m_{1}+m_{2} \geq M}\left\langle\varphi^{T}, v_{m}^{\varepsilon}\right\rangle_{\mathbf{L}^{2}\left(\Omega_{2}\right)} \exp \left[-\left(\left(m_{1}^{2}+m_{2}^{2}\right) \varepsilon+\frac{1}{4 \varepsilon}\right) T\right] u_{m}^{\varepsilon}\right\|_{\mathbf{L}^{2}\left(\Omega_{2}\right)} \leq \frac{\varrho}{3} .
$$

So, using (2.46) and the triangular inequality, we obtain for all $\varepsilon \in\left(\varepsilon_{0} / 2,3 \varepsilon_{0} / 2\right)$ the estimate:

$$
\begin{aligned}
\left\|\varphi^{\varepsilon}(0, \cdot)-\varphi^{\varepsilon_{0}}(0, \cdot)\right\|_{\mathbf{L}^{2}\left(\Omega_{2}\right)} \leq \frac{2 \varrho}{3}+ & \sum_{m_{1}+m_{2}<M} \|\left\langle\varphi^{T}, v_{m}^{\varepsilon}\right\rangle_{\mathbf{L}^{2}\left(\Omega_{2}\right)} \exp \left[-\left(\left(m_{1}^{2}+m_{2}^{2}\right) \varepsilon+\frac{1}{4 \varepsilon}\right) T\right] u_{m}^{\varepsilon} \\
& -\left\langle\varphi^{T}, v_{m}^{\varepsilon_{0}}\right\rangle_{\mathbf{L}^{2}\left(\Omega_{2}\right)} \exp \left[-\left(\left(m_{1}^{2}+m_{2}^{2}\right) \varepsilon_{0}+\frac{1}{4 \varepsilon_{0}}\right) T\right] u_{m}^{\varepsilon_{0}} \|_{\mathbf{L}^{2}\left(\Omega_{2}\right)} .
\end{aligned}
$$

Finally, considering that the sum in the right-hand side of 2.56 is finite and its terms are continuous with respect to $\varepsilon$ (see 2.14 and 2.44 respectively for the continuity of $u_{m}^{\varepsilon}$ and $v_{m}^{\varepsilon}$ ), we have 2.53 for all $\varepsilon \in\left(\varepsilon_{0}-\delta, \varepsilon_{0}+\delta\right)$ if $\delta$ is small enough. 


\section{The cost of the control in $(0, \pi)^{2}$}

In this section we prove the first three items of Theorem 1.1. In the three proofs we use the equivalent definitions of the costs given in (1.4). In addition, for the proof of Item 2 we use the eigenfunctions given in 2.18, whereas for the other two proofs we use Remark 1.5 and then inspire in [20. The three proofs are independent.

\subsection{Proof of Item 1 of Theorem 1.1}

In order to prove that the cost of the control decays, we first state a Carleman inequality for system (1.7), then use that the divergence is null, and finally apply usual parabolic estimates. We recall that $\omega \subset \Omega_{2}$ is any arbitrary subdomain.

We consider $\omega_{0}$ an open ball centered at some point $\bar{x}=\left(\bar{x}_{1}, \bar{x}_{2}\right)$ and whose closure is contained in $\omega$. We consider the auxiliary functions:

$$
\eta^{ \pm}(x):=\left(2 \pi^{2}\right)^{-1}\left({ }_{ \pm} G\left(x_{1}\right)-\left(x_{2}-\bar{x}_{2}\right)^{2}\right),
$$

for $G$ a regular (positive) function such that $G(0)=G(\pi)=0, G^{\prime \prime}<0, G^{\prime}\left(\bar{x}_{1}\right)=0$ and $\|G\|_{L^{\infty}(0, \pi)} \leq \pi^{2}$. Finally, let us consider the following classical weights, for $\tilde{T}>0$ :

$$
\begin{aligned}
& \alpha_{ \pm}(t, x):=\frac{e^{8 \lambda}-e^{\lambda\left(\eta^{ \pm}(x)+6\right)}}{t(\tilde{T}-t)}, \quad \xi(t, x):=\frac{e^{\lambda\left(\eta^{ \pm}(x)+6\right)}}{t(\tilde{T}-t)}, \\
& \alpha_{ \pm}^{*}(t)=\max _{x \in \bar{\Omega}} \alpha_{ \pm}(t, x), \quad \xi_{ \pm}^{*}(t)=\min _{x \in \bar{\Omega}} \xi_{ \pm}(t, x) .
\end{aligned}
$$

Remark 3.1. By the choice of $G$ we have that $\left\|\eta^{ \pm}\right\|_{L^{\infty}\left(\Omega_{2}\right)} \leq 1$, the weights $\alpha^{ \pm}$are positive. This way of defining the weights is classical (see, for instance, [13] and [8]).

Proposition 3.2. There is a constant $C>0$ such that for any domain $\omega \subset \Omega_{2}, \tilde{T}>0$ and $z^{\tilde{T}} \in L^{2}\left(\Omega_{2}\right)$ we have that:

$$
\tau \lambda^{2} \iint_{(0, \tilde{T}) \times \Omega_{2}} e^{-2 \tau \alpha_{+}} \xi_{+}|\nabla z|^{2} d x d t+\tau^{3} \lambda^{4} \iint_{(0, \tilde{T}) \times \Omega_{2}} e^{-2 \tau \alpha_{+}} \xi_{+}^{3}|z|^{2} d x d t \leq C \tau^{3} \lambda^{4} \iint_{(0, \tilde{T}) \times \omega} e^{-2 \tau \alpha_{+}} \xi_{+}^{3}|z|^{2} d x d t,
$$

for $z$ the solution of (1.7) (in $(0, \tilde{T})$ instead of in $(0, T)$ ), for the weights defined in (3.1) and for any $\varepsilon \in(0,1), \lambda \geq C$ and $\tau \geq C\left(\tilde{T}+\tilde{T}^{2}\right) \varepsilon^{-1}$.

The proof of Proposition 3.2 is essentially a combination of the proofs given in [20, Proposition 1] and [12, Lemma 1]. Since the ideas of the proof are not original and since the proof is rather long, we just sketch it in Appendix A,

A straight consequence of Proposition 3.2 and Remark 1.5 is that there is $C>0$ such that for any $\varphi^{T} \in \mathbf{L}^{2}\left(\Omega_{2}\right)$, if $\varphi$ denotes the solution of 1.3$)$ in $\Omega_{2}$, for any $\lambda \geq C$, for any $\tau \geq C \varepsilon^{-1}$, and for the weights 
(3.1) with $\tilde{T}=1$, we have that:

$$
\begin{aligned}
\tau \lambda^{2} \iint_{(0,1) \times \Omega_{2}} e^{-2 \tau \alpha_{+}} \xi_{+}\left|\nabla \varphi_{2}(t+T-1, x)\right|^{2} d x d t+\tau^{3} \lambda^{4} & \iint_{(0,1) \times \Omega_{2}} e^{-2 \tau \alpha_{+}} \xi_{+}^{3}\left|\varphi_{2}(t+T-1, x)\right|^{2} d x d t \\
& \leq C \tau^{3} \lambda^{4} \iint_{(0,1) \times \omega} e^{-2 \tau \alpha_{+}} \xi_{+}^{3}\left|\varphi_{2}(t+T-1, x)\right|^{2} d x d t .
\end{aligned}
$$

Moreover, because of $(1.6)_{2}$, we have by Poincaré inequality (and Fubini) that for all $t<T$ :

$$
\begin{aligned}
& \tau \lambda^{2} \int_{\Omega_{2}} e^{-2 \tau \alpha_{+}^{*}(t)} \xi_{+}^{*}(t)\left|\varphi_{1}\right|^{2}(t, x) d x=\tau \lambda^{2} e^{-2 \tau \alpha_{+}^{*}(t)} \xi_{+}^{*}(t) \int_{0}^{\pi}\left\|\varphi_{1}\left(t, \cdot, x_{2}\right)\right\|_{L^{2}\left(0, \pi ; d x_{1}\right)}^{2} d x_{2} \\
\leq & C \tau \lambda^{2} e^{-2 \tau \alpha_{+}^{*}(t)} \xi_{+}^{*}(t) \int_{0}^{\pi}\left\|\partial_{x_{1}} \varphi_{1}\left(t, \cdot, x_{2}\right)\right\|_{L^{2}\left(0, \pi ; d x_{1}\right)}^{2} d x_{2}=C \tau \lambda^{2} \int_{\Omega_{2}} e^{-2 \tau \alpha_{+}^{*}(t)} \xi_{+}^{*}(t)\left|\partial_{x_{1}} \varphi_{1}(t, x)\right|^{2} d x .
\end{aligned}
$$

Thus, since $\nabla \cdot \varphi=0$, combining 3.3 with 3.4 , we have for $\lambda \geq C, \tau \geq C \varepsilon^{-1}$ the estimate:

$$
\tau \lambda^{2} \iint_{(0,1) \times \Omega_{2}} e^{-2 \tau \alpha_{+}^{*}} \xi_{+}^{*}|\varphi(t+T-1, x)|^{2} d x d t \leq C \tau^{3} \lambda^{4} \iint_{(0,1) \times \omega} e^{-2 \tau \alpha_{+}} \xi_{+}^{3}\left|\varphi_{2}(t+T-1, x)\right|^{2} d x d t .
$$

So, fixing $\lambda$ large enough, and $\tau=\tau_{0} \varepsilon^{-1}$ for $\tau_{0}$ large enough, we have that 3.5 implies that:

$$
\|\varphi\|_{\mathbf{L}^{2}\left((T-2 / 3, T-1 / 3) \times \Omega_{2}\right)} \leq C e^{C \varepsilon^{-1}}\left\|\varphi_{2}\right\|_{L^{2}((T-1, T) \times \omega)} .
$$

Let $T>7$, using Item 2 of Proposition 2.8 there is $C>0$ such that for $\delta=7-2 \pi-2 / 3$, for all $t^{\prime} \in(T-2 / 3, T-1 / 3)$ and for $s=0$, we have that:

$$
\|\varphi(0, \cdot)\|_{\mathbf{L}^{2}\left(\Omega_{2}\right)} \leq C \exp \left[\frac{7-2 / 3-t^{\prime}}{4 \varepsilon}\right]\left\|\varphi\left(t^{\prime}, \cdot\right)\right\|_{\mathbf{L}^{2}\left(\Omega_{2}\right)} \leq C \exp \left[\frac{7-T}{4 \varepsilon}\right]\left\|\varphi\left(t^{\prime}, \cdot\right)\right\|_{\mathbf{L}^{2}\left(\Omega_{2}\right)} .
$$

So, combining (3.6) and (3.7), we get that, for any $T>7$ :

$$
\|\varphi(0, \cdot)\|_{\mathbf{L}^{2}\left(\Omega_{2}\right)} \leq C \exp \left[\frac{7-T}{4 \varepsilon}\right]\|\varphi\|_{\mathbf{L}^{2}\left((T-2 / 3, T-1 / 3) \times \Omega_{2}\right)} \leq C \exp \left[\frac{C-T}{4 \varepsilon}\right]\left\|\varphi_{2}\right\|_{L^{2}((T-1, T) \times \omega)} .
$$

In particular, if $T_{0}$ is sufficiently large, Item 1 of Theorem 1.1 is true.

\subsection{Proof of Item 2 of Theorem 1.1}

Let us fix $h \in(0, \pi)$ and a control domain $\omega \subset(0, \pi) \times(\pi-h, \pi)$. We prove that, for $T \in(0,2(\pi-h))$ fixed, the cost of the control is at least exponentially large with respect to $\varepsilon^{-1}$. We recall that:

$$
u(x):=c e^{-(2 \varepsilon)^{-1} x_{2}}\left(\sin \left(x_{1}\right)\left(2 \varepsilon \cos \left(x_{2}\right)-\sin \left(x_{2}\right)\right),-2 \varepsilon \cos \left(x_{1}\right) \sin \left(x_{2}\right)\right),
$$

for:

$$
c:=\sqrt{\frac{1+4 \varepsilon^{2}}{\left(1-e^{-\pi \varepsilon^{-1}}\right) \pi \varepsilon^{3}\left(1+8 \varepsilon^{2}\right)}},
$$

is a solution of 1.5 for $\lambda_{\varepsilon}:=2 \varepsilon+\frac{1}{4 \varepsilon}$ and such that $\|u\|_{\mathbf{L}^{2}\left(\Omega_{2}\right)}=1$ (see Proposition 2.5). We remark that since $\omega \subset(0, \pi) \times(\pi-h, \pi)$ we have for all $\delta>0$ and $\varepsilon \in(0,1)$ the estimate:

$$
\|u\|_{\mathbf{L}^{\infty}(\omega)} \leq C \varepsilon^{-3 / 2} e^{-(2 \varepsilon)^{-1}(\pi-h)} \leq C_{\delta} e^{-(2 \varepsilon)^{-1}(\pi-h-\delta)} .
$$

Indeed, we have that $\varepsilon^{-3} \leq C_{\delta} e^{\delta \varepsilon^{-1}}$ for all $\delta>0$ and $\varepsilon \in \mathbb{R}^{+}$. 
For proving (3.8) we have calculated the maximum of $e^{-2 \varepsilon^{-1} x_{2}}$ in $(0, \pi) \times(\pi-h, \pi)$. Moreover, we have that:

$$
\varphi(t, x):=u(x) e^{-\lambda_{\varepsilon}(T-t)},
$$

is a solution of 1.3 in $\Omega_{2}$ for $\varphi^{T}=u$. On the one hand, we have that:

$$
\int_{\Omega_{2}}|\varphi(0, x)|^{2} d x=e^{-2 \lambda_{\varepsilon} T}
$$

On the other hand, using (3.8) and bounding $e^{-2 \lambda_{\varepsilon}(T-t)}$ by 1 we obtain the estimate:

$$
\iint_{Q_{\omega}}|\varphi|^{2} d x d t=\iint_{Q_{\omega}}|u(x)|^{2} e^{-2 \lambda_{\varepsilon}(T-t)} d x d t \leq\|u\|_{\mathbf{L}^{\infty}(\omega)}^{2} \iint_{Q_{\omega}} d x d t \leq C_{\delta} \exp \left[\frac{h-\pi+\delta}{\varepsilon}\right] .
$$

So, combining (1.4), (3.9) and 3.10 we get that:

$$
\left[K_{1}(T, \varepsilon, \omega)\right]^{2} \geq c_{\delta} \exp \left[\frac{\pi-h-\delta-4 \varepsilon^{2} T-T / 2}{\varepsilon}\right],
$$

which implies Item 2 of Theorem 1.1 by choosing $\delta \in(0, \pi-h-T / 2)$.

\subsection{Proof of Item 3 of Theorem 1.1}

Let $h \in(0, \pi)$, a control domain $\omega \subset(0, \pi) \times(0, h)$ and $T \in(0, \pi-h)$ fixed. In order to prove that the cost of the control blows out exponentially with respect to $\varepsilon$, we shall adapt the proof given in 20 , Theorem 1]. Indeed, unlike in [20], $\varphi$ is a vectorial function, the boundary conditions are not purely Dirichlet, and we cannot pick in 1.3 an initial value $\varphi^{T}$ such that $\int_{\Omega} \varphi_{2}^{T}>0$ because for all $u \in \mathcal{H}(\Omega)$ we have that $\int_{\Omega} u_{1}=\int_{\Omega} u_{2}=0$.

To make the proof more clear, we split it with the help of two lemmas:

Lemma 3.3. Let $h \in(0, \pi), T \in(0, \pi-h)$ and $\delta \in(0, \pi-T-h)$. Let $\varphi^{T} \in \mathcal{H}\left(\Omega_{2}\right) \cap \mathbf{C}^{\infty}\left(\overline{\Omega_{2}}\right) \backslash\{0\}$ such that $\operatorname{supp}\left(\varphi^{T}\right) \subset(0, \pi) \times(\pi-\delta, \pi)$ and let $\varphi^{\varepsilon}$ be the solution of 1.3) in $\Omega_{2}$ and with initial value $\varphi^{T}$. Then, there is $c>0$ such that, for all $\varepsilon \in(0,1)$ :

$$
\left\|\varphi^{\varepsilon}(0, \cdot)\right\|_{\mathbf{L}^{2}(\Omega)} \geq c .
$$

Remark 3.4. For any $\delta>0$ there are functions $\varphi^{T}$ which satisfy the hypothesis of Lemma 3.3 For example, if $\chi_{\delta} \in C^{\infty}([0, \pi])$ is a function supported in $(\pi-\delta, \pi)$ of null mean, we can consider:

$$
\varphi^{T}(x):=\left(\int_{0}^{x_{1}} \chi_{\delta}(s) d s \chi_{\delta}\left(x_{2}\right),-\chi_{\delta}\left(x_{1}\right) \int_{0}^{x_{2}} \chi_{\delta}(s) d s\right) .
$$

Consequently, neither Lemma 3.3 nor Lemma 3.5 below are empty results.

Proof. We prove (3.11) by contradiction. First of all, we remark that, thanks to forward uniqueness of (1.3) (which can be proved by contradiction with backward uniqueness and $(2.46),\left\|\varphi^{\varepsilon}(0, \cdot)\right\|_{\mathbf{L}^{2}(\Omega)}>0$ for all $\varepsilon \in(0,1]$. In addition, because of Remark 2.9, the only problematic situation arises if there is $\varepsilon_{k} \rightarrow 0$ such that $\left\|\varphi^{\varepsilon_{k}}(0, \cdot)\right\|_{\mathbf{L}^{2}(\Omega)} \rightarrow 0$. Let us suppose the existence of such sequence $\varepsilon_{k}$ and derive a contradiction. 
We have that $\left\{\varphi_{2}^{\varepsilon}: \varepsilon \in(0,1)\right\}$ is bounded in $L^{2}(Q)$ (see Remark 1.5); thus, we can suppose that $\varphi_{2}^{\varepsilon_{k}}$ converges weakly in $L^{2}(Q)$ to some function $\gamma$. Let us consider $\psi \in \mathcal{D}\left([0, T] \times \Omega_{2}\right)$. Using Remark 1.5 we have that:

$$
0=\iint_{Q} \varphi_{2}^{\varepsilon_{k}}\left(\partial_{t} \psi-\varepsilon_{k} \Delta \psi+\partial_{x_{2}} \psi\right) d x d t+\left\langle\varphi_{2}^{\varepsilon_{k}}(0, \cdot), \psi(0, \cdot)\right\rangle_{L^{2}\left(\Omega_{2}\right)}-\left\langle\varphi_{2}^{T}, \psi(T, \cdot)\right\rangle_{L^{2}\left(\Omega_{2}\right)} .
$$

By taking the weak limit of $\varphi_{2}^{\varepsilon_{k}}$, the strong limit of $\varepsilon_{k} \Delta \psi$ (to 0 ) and the strong limit of $\varphi_{2}^{\varepsilon_{k}}(0, \cdot)$ (to 0 ), we get from 3.13 that:

$$
0=\iint_{Q} \gamma\left(\partial_{t} \psi+\partial_{x_{2}} \psi\right) d t d x-\left\langle\varphi_{2}^{T}, \psi(T, \cdot)\right\rangle_{L^{2}\left(\Omega_{2}\right)} .
$$

We have that equation 3.14 is true in particular for $\psi(t, x)=\varphi_{2}^{T}\left(x_{1}, x_{2}+T-t\right)$, which belongs to $\mathcal{D}([0, T] \times \Omega)$. Consequently, we get that $\left\|\varphi_{2}^{T}\right\|_{L^{2}\left(\Omega_{2}\right)}=0$. Since $\varphi^{T} \in \mathcal{H}\left(\Omega_{2}\right) \cap \mathbf{C}^{1}\left(\overline{\Omega_{2}}\right)$ this implies that $\varphi_{1}^{T}=0$, contradicting the fact that $\varphi^{T} \neq 0$.

Lemma 3.5. Let $h \in(0, \pi), T \in(0, \pi-h)$ and $\delta \in(0, \pi-T-h)$. Then, if $\varphi^{T} \in \mathcal{H}\left(\Omega_{2}\right)$ such that $\operatorname{supp}\left(\varphi^{T}\right) \subset(0, \pi) \times(\pi-\delta, \pi)$ there is $c>0$ such that for all $\varepsilon \in(0,1)$ :

$$
\iint_{Q_{\omega}}\left|\varphi_{2}^{\varepsilon}\right|^{2} d x d t \leq c e^{-c \varepsilon^{-1}}
$$

for $\varphi^{\varepsilon}$ the solution of 1.3 in $\Omega_{2}$ and for initial value $\varphi^{T}$.

For the following proof we fix a parameter $\tilde{\delta}>0$ so that $T \in\left(0, \frac{\pi-h-\delta}{1+\tilde{\delta}}\right)$.

Proof. First of all, we recall that $\varphi_{2}^{\varepsilon}$ satisfies 1.7 for $z^{T}:=\varphi_{2}^{T}$. Multiplying 1.7$)_{1}$ by

$$
2 e^{2\left(\tilde{\delta}\left(\pi-x_{2}\right)-\left(\tilde{\delta}+(\tilde{\delta})^{2}\right)(T-t)\right) \varepsilon^{-1}} \varphi_{2}^{\varepsilon},
$$

and integrating in $\Omega_{2}$ we deduce that for all $t \in[0, T]$ we have the following equality:

$$
-\frac{d}{d t}\left(\int_{\Omega_{2}}\left|e^{\left(\tilde{\delta}\left(\pi-x_{2}\right)-\left(\tilde{\delta}+(\tilde{\delta})^{2}\right)(T-t)\right) \varepsilon^{-1}} \varphi_{2}^{\varepsilon}\right|^{2} d x\right)+2 \varepsilon \int_{\Omega_{2}}\left|\nabla\left(e^{\left(\tilde{\delta}\left(\pi-x_{2}\right)-\left(\tilde{\delta}+(\tilde{\delta})^{2}\right)(T-t)\right) \varepsilon^{-1}} \varphi_{2}^{\varepsilon}\right)\right|^{2} d x=0 .
$$

Indeed, first, we have the equality:

$$
\begin{aligned}
-2 \int_{\Omega_{2}} e^{2\left(\tilde{\delta}\left(\pi-x_{2}\right)-\left(\tilde{\delta}+(\tilde{\delta})^{2}\right)(T-t)\right) \varepsilon^{-1}} \varphi_{2}^{\varepsilon} \partial_{t} \varphi_{2}^{\varepsilon} d x & =-\frac{d}{d t}\left(\int_{\Omega_{2}}\left|e^{\left(\tilde{\delta}\left(\pi-x_{2}\right)-\left(\tilde{\delta}+(\tilde{\delta})^{2}\right)(T-t)\right) \varepsilon^{-1}} \varphi_{2}^{\varepsilon}\right|^{2} d x\right) \\
+ & 2\left(\tilde{\delta}+(\tilde{\delta})^{2}\right) \varepsilon^{-1} \int_{\Omega_{2}} e^{2\left(\tilde{\delta}\left(\pi-x_{2}\right)-\left(\tilde{\delta}+(\tilde{\delta})^{2}\right)(T-t)\right) \varepsilon^{-1}}\left|\varphi_{2}^{\varepsilon}\right|^{2} d x .
\end{aligned}
$$

Second, since $\varphi_{2}^{\varepsilon}$ satisfies $(1.7)_{2}$ and $(1.7)_{3}$ we have that:

$$
\begin{aligned}
& -2 \varepsilon \int_{\Omega_{2}} e^{2\left(\tilde{\delta}\left(\pi-x_{2}\right)-\left(\tilde{\delta}+(\tilde{\delta})^{2}\right)(T-t)\right) \varepsilon^{-1}} \varphi_{2}^{\varepsilon} \Delta \varphi_{2}^{\varepsilon} d x \\
& \quad=2 \varepsilon \int_{\Omega_{2}} e^{2\left(\tilde{\delta}\left(\pi-x_{2}\right)-\left(\tilde{\delta}+(\tilde{\delta})^{2}\right)(T-t)\right) \varepsilon^{-1}}\left|\nabla \varphi_{2}^{\varepsilon}\right|^{2} d x-4 \tilde{\delta} \int_{\Omega_{2}} e^{2\left(\tilde{\delta}\left(\pi-x_{2}\right)-\left(\tilde{\delta}+(\tilde{\delta})^{2}\right)(T-t)\right) \varepsilon^{-1}} \partial_{x_{2}} \varphi_{2}^{\varepsilon} \varphi_{2}^{\varepsilon} d x \\
& =2 \varepsilon \int_{\Omega_{2}}\left|\nabla\left(e^{\left(\tilde{\delta}\left(\pi-x_{2}\right)-\left(\tilde{\delta}+(\tilde{\delta})^{2}\right)(T-t)\right) \varepsilon^{-1}} \varphi_{2}^{\varepsilon}\right)\right|^{2} d x-2(\tilde{\delta})^{2} \varepsilon^{-1} \int_{\Omega_{2}} e^{2\left(\tilde{\delta}\left(\pi-x_{2}\right)-\left(\tilde{\delta}+(\tilde{\delta})^{2}\right)(T-t)\right) \varepsilon^{-1}}\left|\varphi_{2}^{\varepsilon}\right|^{2} d x .
\end{aligned}
$$

Thirdly, since $\varphi_{2}^{\varepsilon}$ satisfies $[1.7]_{3}$ we have the equality:

$$
-2 \int_{\Omega_{2}} e^{2\left(\tilde{\delta}\left(\pi-x_{2}\right)-\left(\tilde{\delta}+(\tilde{\delta})^{2}\right)(T-t)\right) \varepsilon^{-1}} \varphi_{2}^{\varepsilon} \partial_{x_{2}} \varphi_{2}^{\varepsilon} d x=-2 \tilde{\delta} \varepsilon^{-1} \int_{\Omega_{2}} e^{2\left(\tilde{\delta}\left(\pi-x_{2}\right)-\left(\tilde{\delta}+(\tilde{\delta})^{2}\right)(T-t)\right) \varepsilon^{-1}}\left|\varphi_{2}^{\varepsilon}\right|^{2} d x .
$$

Consequently, considering (1.7) ${ }_{1}$ and (3.17)- 3.19) we obtain (3.16). 
So, we obtain from $(3.16)$ the following Agmon identity:

$$
\begin{aligned}
& \int_{0}^{T} \int_{\Omega_{2}}\left|e^{\left(\tilde{\delta}\left(\pi-x_{2}\right)-\left(\tilde{\delta}+(\tilde{\delta})^{2}\right)(T-t)\right) \varepsilon^{-1}} \varphi_{2}^{\varepsilon}\right|^{2} d x d t \\
& \quad+2 \varepsilon \int_{0}^{T} \int_{t}^{T} \int_{\Omega_{2}}\left|\nabla\left(e^{\left(\tilde{\delta}\left(\pi-x_{2}\right)-\left(\tilde{\delta}+(\tilde{\delta})^{2}\right)(T-s)\right) \varepsilon^{-1}} \varphi_{2}^{\varepsilon}(s, x)\right)\right|^{2} d x d s d t=T \int_{\Omega_{2}}\left|e^{\tilde{\delta}\left(\pi-x_{2}\right) \varepsilon^{-1}} \varphi_{2}^{T}\right|^{2} d x .
\end{aligned}
$$

On the one hand, using that the support of $\varphi^{T}$ is included in $(0, \pi) \times(\pi-\delta, \pi)$, we have that:

$$
T \int_{\Omega_{2}}\left|e^{\tilde{\delta}\left(\pi-x_{2}\right) \varepsilon^{-1}} \varphi_{2}^{T}\right|^{2} d x \leq \pi e^{2 \tilde{\delta} \delta \varepsilon^{-1}} \int_{\Omega_{2}}\left|\varphi_{2}^{T}\right|^{2} d x .
$$

On the other hand, recalling that $\omega \subset(0, \pi) \times(0, h)$, we find that:

$$
e^{2 \tilde{\delta}(\pi-h-T-\tilde{\delta} T) \varepsilon^{-1}} \iint_{Q_{\omega}}\left|\varphi_{2}^{\varepsilon}\right|^{2} d x d t \leq \int_{0}^{T} \int_{\Omega_{2}}\left|e^{\left(\tilde{\delta}\left(\pi-x_{2}\right)-\left(\tilde{\delta}+(\tilde{\delta})^{2}\right)(T-t)\right) \varepsilon^{-1}} \varphi_{2}^{\varepsilon}\right|^{2} d x d t .
$$

Combining 3.20, 3.21 and 3.22, we have that:

$$
\iint_{Q_{\omega}}\left|\varphi_{2}^{\varepsilon}\right|^{2} d x d t \leq \pi \exp \left[\frac{2 \tilde{\delta}(T+\tilde{\delta} T+h+\delta-\pi)}{\varepsilon}\right]\left\|\varphi_{2}^{T}\right\|_{L^{2}(\Omega)}^{2} ;
$$

that is, we get the decay of the $L^{2}$ norm of $\varphi_{2}$ in the control domain.

Remark 3.6. So far, combining (1.4), Lemma 3.3. Lemma 3.5 and Remark 3.4 we have that for a given $h \in(0, \pi)$, for all $\omega \subset(0, T) \times(0, h)$, and for all $T \in(0, \pi-h)$, there is $c>0$ such that for all $\varepsilon \in(0,1)$ :

$$
K_{2}(T, \varepsilon, \omega) \geq c e^{c \varepsilon^{-1}} \text {. }
$$

Now we have the tools to end the proof of Item 3 of Theorem 1.1. For that proof, we denote, for $h \in(0, \pi)$ and $T \in(0, \pi-h)$ both fixed parameters:

$$
\delta:=(\pi-h-T) / 3, \quad \omega_{1}:=(0, \pi) \times(0, h), \quad \omega_{2}:=(0, \pi) \times(0, h+\delta) .
$$

End of the proof of Item 3 of Theorem 1.1. Let $\varphi^{\varepsilon}$ be the solution of 1.3 in $\Omega_{2}$ of initial value (3.12) and with $\delta$ as in (3.24). Thanks to 1.4, Lemma 3.3 and Lemma 3.5, the only thing left to prove is that $\iint_{Q_{\omega}}\left|\varphi_{1}^{\varepsilon}\right|^{2} d x d t$ decays exponentially. For that purpose, we get an estimate of $\partial_{x_{2}} \varphi_{2}^{\varepsilon}$ in $L^{2}\left(Q_{\omega_{1}}\right)$.

Let us fix $\theta(s)$ a positive regular scalar cut-off function supported in $(-\infty, h+\delta)$ and such that $\theta=1$ in $(-\infty, h]$. We define:

$$
\psi^{\varepsilon}(t, x):=\theta\left(x_{2}\right) \varphi_{2}^{\varepsilon}(t, x) .
$$

Recalling the support of $\varphi^{T}$ (see 3.12 ), we have that $\psi^{\varepsilon}$ satisfies:

$$
\begin{cases}-\partial_{t} \psi^{\varepsilon}-\varepsilon \Delta \psi^{\varepsilon}-\partial_{x_{2}} \psi^{\varepsilon}=\varepsilon \theta^{\prime \prime}\left(x_{2}\right) \varphi_{2}^{\varepsilon}-2 \varepsilon \partial_{x_{2}}\left(\theta^{\prime}\left(x_{2}\right) \varphi_{2}^{\varepsilon}\right)-\theta^{\prime}\left(x_{2}\right) \varphi_{2}^{\varepsilon} & \text { in } Q \\ \psi^{\varepsilon}=0 & \text { on } \Sigma_{b, t} \\ \partial_{x_{1}} \psi^{\varepsilon}=0 & \text { on } \Sigma_{l, r} \\ \psi^{\varepsilon}(T, \cdot)=0 & \text { on } \Omega_{2}\end{cases}
$$


In particular, if we multiply $(3.26)$ by $\psi^{\varepsilon}$ and we integrate by parts, it is not difficult to deduce that:

$$
\iint_{Q}\left|\nabla \psi^{\varepsilon}\right|^{2} d x d t \leq \frac{C}{\varepsilon} \iint_{Q_{\omega_{2}}}\left|\varphi_{2}^{\varepsilon}\right|^{2} d x d t
$$

Using Lemma 3.5 for $h^{\prime}=h+\delta, T^{\prime}=T$ and $\delta^{\prime}=\delta$ and using that for all $\tilde{\delta}>0$ there is $C_{\tilde{\delta}}>0$ such that $\varepsilon^{-1} \leq C_{\tilde{\delta}} e^{\tilde{\delta} \varepsilon^{-1}}$ in $\mathbb{R}^{+}$, we get from 3.27 that:

$$
\iint_{Q_{\omega_{1}}}\left|\partial_{x_{2}} \varphi_{2}^{\varepsilon}\right|^{2} d x d t \leq C e^{-c \varepsilon^{-1}}
$$

In order to conclude, using that the divergence of $\varphi^{\varepsilon}$ is null, we get that:

$$
\iint_{Q_{\omega_{1}}}\left|\partial_{x_{1}} \varphi_{1}^{\varepsilon}\right|^{2} d x d t \leq C e^{-c \varepsilon^{-1}}
$$

In addition, since $\varphi_{1}^{\varepsilon}$ is null on $x_{1}=0$ (see 1.6$)$ ), we have, thanks to Poincaré inequality:

$$
\iint_{Q_{\omega}}\left|\varphi_{1}^{\varepsilon}\right|^{2} d x d t \leq \iint_{Q_{\omega_{1}}}\left|\varphi_{1}^{\varepsilon}\right|^{2} d x d t \leq C e^{-c \varepsilon^{-1}} .
$$

So, combining (1.4, 3.11, 3.15) and (3.28) we end the proof of Item 3 of Theorem 1.1 .

\section{The control problem $(\mathbf{1 . 1})$ in $(0, \pi)^{3}$}

In this section we first give some solutions of $(1.5)$ in $\Omega_{3}$ and then, we prove Item 4 of Theorem 1.1

\subsection{Brief study of the spectral decomposition of $\mathcal{H}\left((0, \pi)^{3}\right)$}

In order to do the spectral decomposition, we consider the set of separated variables with null divergence, null normal trace, and a null component:

$$
\begin{aligned}
S V & \left(\Omega_{3}\right):=S V_{1}\left(\Omega_{3}\right) \cup S V_{2}\left(\Omega_{3}\right) \cup S V_{3}\left(\Omega_{3}\right):= \\
& \left\{\left(0, g_{1}\left(x_{1}\right) \int_{0}^{x_{2}} g_{2}(s) d s g_{3}\left(x_{3}\right),-g_{1}\left(x_{1}\right) g_{2}\left(x_{2}\right) \int_{0}^{x_{3}} g_{3}(s) d s\right): g_{1} \in L^{2}(0, \pi) ; g_{2}, g_{3} \in L_{0}^{2}(0, \pi)\right\} \\
\cup & \left\{\left(-\int_{0}^{x_{1}} g_{1}(s) d s g_{2}\left(x_{2}\right) g_{3}\left(x_{3}\right), 0, g_{1}\left(x_{1}\right) g_{2}\left(x_{2}\right) \int_{0}^{x_{3}} g_{3}(s) d s\right): g_{2} \in L^{2}(0, \pi) ; g_{1}, g_{3} \in L_{0}^{2}(0, \pi)\right\} \\
& \cup\left\{\left(\int_{0}^{x_{1}} g_{1}(s) d s g_{2}\left(x_{2}\right) g_{3}\left(x_{3}\right),-g_{1}\left(x_{1}\right) \int_{0}^{x_{2}} g_{2}(s) d s g_{3}\left(x_{3}\right), 0\right): g_{3} \in L^{2}(0, \pi) ; g_{1}, g_{2} \in L_{0}^{2}(0, \pi)\right\} .
\end{aligned}
$$

Remark 4.1. Unlike in $S V\left(\Omega_{2}\right)$, now the mean of one of the three auxiliary functions is not necessarily 0 . This difference is crucial in explaining why the solutions of 1.3 behaves differently in $\Omega_{3}$. Indeed, this difference allows us to construct eigenfunctions whose associated eigenvalue does not explode with $\varepsilon$ (see 4.2 ), which is something that we cannot do in $\Omega_{2}$.

In order to look for eigenfunctions in $S V\left(\Omega_{3}\right)$, we remark that if $g_{1}, g_{2}, g_{3}$ are regular functions, the boundary conditions of 1.3 translates into:

$$
g_{1}^{\prime}(0)=g_{1}^{\prime}(\pi)=g_{2}^{\prime}(0)=g_{2}^{\prime}(\pi)=\varepsilon g_{3}^{\prime}(0)+g_{3}(0)=\varepsilon g_{3}^{\prime}(\pi)+g_{3}(\pi)=0 .
$$


Let us focus on $S V_{3}\left(\Omega_{3}\right)$. We can again consider for $m_{1}, m_{2} \in \mathbb{N}_{*}, g_{1, m_{1}}(s):=\cos \left(m_{1} s\right)$ and $g_{2, m_{2}}(s):=$ $\cos \left(m_{2} s\right)$. As for $g_{3}$, we can look at the solutions of:

$$
g^{\prime \prime}+\frac{g^{\prime}}{\varepsilon}+\left(\frac{\lambda}{\varepsilon}-m_{1}^{2}-m_{2}^{2}\right) g=0,
$$

an equation that can be obtained as in the proof of Proposition 2.5. Since the mean of $g_{3}$ is not necessarily 0 and since $\int_{0}^{s} g_{3}\left(s^{\prime}\right) d s^{\prime}$ does not have to be an eigenfunction of $-\varepsilon \Delta+\partial_{x_{3}}$ (because the third component of an element of $S V_{3}\left(\Omega_{3}\right)$ is null), we can consider small values of $\lambda$. In particular we get for any $m_{1}, m_{2} \in \mathbb{N}_{*}$ the following eigenfunction:

$$
\left(\frac{1}{m_{1}} \sin \left(m_{1} x_{1}\right) \cos \left(m_{2} x_{2}\right) e^{-\varepsilon^{-1} x_{3}},-\frac{1}{m_{2}} \cos \left(m_{1} x_{1}\right) \sin \left(m_{2} x_{2}\right) e^{-\varepsilon^{-1} x_{3}}, 0\right),
$$

whose associated eigenvalues is:

$$
\lambda_{m_{1}, m_{2}}^{\varepsilon}:=\varepsilon\left(m_{1}^{2}+m_{2}^{2}\right),
$$

and whose associated pressure term is constant.

Remark 4.2. We can get for dimension 3 similar results to those of Remark 1.4 Proposition 2.1, Proposition 2.5 and Proposition 2.7, with the only difference that some eigenvalues do not explode with $\varepsilon$. In that sense, we can get similar results as Item 1 of Proposition 2.8, but we cannot get a general dissipation result as the one in Item 2 of Proposition 2.8 .

\subsection{Proof of Item 4 of Theorem 1.1}

For this proof we consider the function:

$$
u(x):=\frac{2 e^{-\varepsilon^{-1} x_{3}}}{\pi \sqrt{\varepsilon\left(1-e^{-2 \pi \varepsilon^{-1}}\right)}}\left(\sin \left(x_{1}\right) \cos \left(x_{2}\right),-\cos \left(x_{1}\right) \sin \left(x_{2}\right), 0\right),
$$

which by (4.2) and 4.3 (we have taken $m_{1}=m_{2}=1$ ) is a solution of 1.5) in $\Omega_{3}$ for $\lambda=2 \varepsilon$ and a pressure which is constant. Moreover, we can easily check that $\|u\|_{\mathbf{L}^{2}(\Omega)}=1$ and that $\varphi(t, x):=u(x) e^{-2 \varepsilon(T-t)}$ is a solution of 1.3 in $\Omega_{3}$. In addition, since $\omega \subset(0, \pi)^{2} \times(\pi-h, \pi)$ we can bound $e^{-\varepsilon^{-1} x_{3}}$ by $e^{-(\pi-h) \varepsilon^{-1}}$ and obtain for all $\delta>0$ and $\varepsilon \in(0,1)$ that:

$$
\|u\|_{\mathbf{L}^{\infty}(\omega)} \leq C \varepsilon^{-1 / 2} e^{-(\pi-h) \varepsilon^{-1}} \leq C_{\delta} e^{-\varepsilon^{-1}(\pi-h-\delta)} .
$$

Indeed, we have that $\varepsilon^{-1 / 2} \leq C_{\delta} e^{\delta \varepsilon^{-1}}$ for all $\delta>0$ and $\varepsilon \in \mathbb{R}^{+}$.

On the one hand, we have that:

$$
\int_{\Omega_{3}}|\varphi(0, x)|^{2} d x=e^{-4 \varepsilon T} .
$$

On the other hand, using 4.4 and bounding $e^{-2 \varepsilon(T-t)}$ by 1 we obtain that:

$$
\iint_{Q_{\omega}}|\varphi|^{2} d x d t=\iint_{Q_{\omega}}|u(x)|^{2} e^{-4 \varepsilon(T-t)} d x d t \leq\|u\|_{\mathbf{L}^{\infty}(\omega)}^{2} \iint_{Q_{\omega}} d x d t \leq T C_{\delta} \exp \left[\frac{2(-\pi+h+\delta)}{\varepsilon}\right] .
$$


So, if we combine (4.5) and 4.6), we have that for all $\delta \in(0, \pi-h)$ (remember 1.4 ) and that $\varepsilon \in(0,1)$ ):

$$
\left[K_{3}(T, \varepsilon, \omega)\right]^{2} \geq \frac{\int_{\Omega_{3}}|\varphi(0, x)|^{2} d x}{\iint_{Q_{\omega}}|\varphi|^{2} d x d t} \geq c_{\delta} \frac{e^{-4 T}}{T} \exp \left[\frac{2(\pi-h-\delta)}{\varepsilon}\right] ;
$$

that is, for $T$ fixed the cost of the control explodes exponentially when $\varepsilon \rightarrow 0^{+}$.

Remark 4.3. A difference between dimension 3 and dimension 2 can be seen by comparing (3.9) and (4.5). Indeed, for $T$ fixed $(3.9)$ decays with $\varepsilon$, whereas 4.5 does not.

\section{$5 \quad$ Further comments and open problems}

- About the boundary control. The case where we have a control on the boundary of 1.1) (as proposed in [7]) instead of in the interior remains an open problem.

- The spectral method in the transport-diffusion elemental equation. Let us consider the control problem studied in [9, 20]:

$$
\begin{cases}y_{t}+\mathcal{L}^{\varepsilon} y=1_{\omega} f & \text { in } Q \\ y=0 & \text { on } \Sigma \\ y(0, \cdot)=y^{0} & \text { on } \Omega\end{cases}
$$

for $\mathcal{L}^{\varepsilon}:=-\varepsilon \Delta+\partial_{x_{d}}$, for $\Omega$ any open regular domain. In order to prove the dissipation result for the adjoint system, we can use a spectral decomposition. In particular, if $\left\{w_{m}\right\}$ is the spectral basis when we diagonalize the Dirichlet Laplacian in $\Omega$, of eigenvalues $\lambda_{m}$, for $\tilde{w}_{m}:=w_{m} e^{(2 \varepsilon)^{-1} x_{d}}$ we have that $\left\{\tilde{w}_{m}\right\}$ is a linearly independent total set such that $\mathcal{L}^{\varepsilon} \tilde{w}_{m}=\left(\varepsilon \lambda_{m}+(4 \varepsilon)^{-1}\right) \tilde{w}_{m}$, and such that each $\tilde{w}_{m}$ satisfies Dirichlet boundary conditions. Consequently, we can replicate the procedure of Section 2.1 and get the dissipation result, with an accurate result (see (2.47)), which looking at the eigenvalues clearly is the optimal one. As for the optimal dissipation rate, there is another proof in [9, Lemma 4] which uses a representation theorem and a comparison theorem.

- The control problem (1.1) when we change the boundary conditions. We can attempt for $\Omega=(0, \pi)^{2}$ and $d=2$ to replicate this method to get similar results with a boundary condition of the type:

$$
\left(-2 \varepsilon D u \cdot n+\gamma n_{2} u\right)_{\mathrm{tg}}=0
$$

for $\gamma \in \mathbb{R}$ (it is specially interesting the case $\gamma=1 / 2$, when the operator is self-adjoint). Even if it actually produces eigenfunctions, it does it by taking into account the other two roots of 2.22); that is, $r= \pm m_{1}$. In particular, we get eigenfunctions with $g_{2, m_{2}}$ of the type (see 2.24)):

$$
A \cos \left(m_{2} \pi s\right) e^{(2 \varepsilon)^{-1} s}+B \sin \left(m_{2} \pi s\right) e^{(2 \varepsilon)^{-1} s}+C e^{m_{1} s}+D e^{-m_{1} s},
$$

and with $m_{2}$ not necessarily in $\mathbb{N}_{*}$ (and not necessarily easy to calculate explicitly). The root $r=m_{1}$ does not depend on $\varepsilon$ or, more astonishingly, on $\lambda$, because:

$$
u\left(x_{1}, x_{2}\right):=\left(\sin \left(m_{1} x_{1}\right) e^{m_{1} x_{2}},-\cos \left(m_{1} x_{1}\right) e^{m_{1} x_{2}}\right)=\nabla\left(\frac{-\cos \left(m_{1} x_{1}\right) e^{m_{1} x_{2}}}{m_{1}}\right) .
$$


Therefore, that term is an eigenfunction independent of $m_{2}$ or $\varepsilon$ because of the help of the pressure term. A similar thing happens with the term of $D e^{-m_{1} s}$. All this, of course, adds some difficulties in proving that they form a total set (if they actually do), difficulties which we have been unable to overcome. Therefore, getting the eigenfunctions of the Stokes system with a boundary condition of the type (5.2) (together with $u \cdot n=0$ ) can be considered for future work.

As for the control problem (1.1) with Dirichlet boundary conditions, replicating the method of this paper has several problems. For instance, in dimension 2, using the notation of 2.1], we now have the boundary conditions:

$$
g_{1}(0)=g_{1}(\pi)=g_{2}(0)=g_{2}(\pi)=0 .
$$

So, the analogous choice for $g_{1, m_{1}}$ would be $\sin \left(m_{1} s\right)$ (see 2.15$)_{1}$ ); but, for $m_{1}$ odd it does not belong to $L_{0}^{2}(0, \pi)$. Moreover, for any $m_{1} \in \mathbb{N}_{*}, \int_{0}^{s} \sin \left(m_{1} s^{\prime}\right) d s^{\prime}=m_{1}^{-1}\left(1-\cos \left(m_{1} s\right)\right)$, which is not an eigenfunction of the Laplacian. Consequently, it is very likely that another method must be used to get the cost of the control.

- The control problem $(1.1)$ in other domains. It is clear that working in $(0, \pi)^{2}$ or $(0, \pi)^{3}$ is really helpful, not only for the dissipation, but for the cost of the control as well. Indeed, we have used several times in Section 3 that the solutions of 1.3 are solutions of the heat equation. Consequently, it would be interesting to know what results can be obtained in other domains.

- The cost of the control in dimension 3 if $\omega$ is near $x_{3}=0$. It is an open question if $K_{3}(T, \varepsilon, \omega)$ (see 1.2 ) also explodes with $\varepsilon$ for large times $T$ if there is $\bar{x} \in(0, \pi)^{2} \times\{0\}$ and $\delta>0$ such that $B(\bar{x}, \delta) \cap\left\{x_{3}>0\right\} \subset \omega$.

- The optimal $T_{0}$ in which the cost of the control decays with $\varepsilon$. The optimal $T_{0}$ in which the cost of the control decays with $\varepsilon$ is an open problem (see Item 1 of Theorem 1.1). Thanks to Item 2 and Item 3 of Theorem 1.1 we can get a lower bound. Moreover, we could have got an upper bound by doing all the operations of the proof of the Carleman (Proposition 3.2 explicitly as in 9 , but it would be far from optimal, so is not worth the effort. A more subtle technique would be to transform the problem in a fast-control problem as in [25]; but, on the one hand, when we multiply by a weight which depends on a spatial variable, the fact that the divergence is null is lost; and, on the other hand, the fact that fast controls have a cost of $e^{C T^{-1}}$ (without specifying the $C$ ) in the Stokes system is just a recent result (see [4]). In addition, this problem is probably more difficult than getting the optimal $T_{0}$ for (5.1), which is still an open problem, even in dimension 1.

- Relation of the cost of control with a force of $d-1$ components and with a force of $d$ components. An interesting question is to see if the fact that $K_{1}(T, \varepsilon, \omega)$ decays or explodes with $\varepsilon$ is equivalent to the fact that $K_{2}(T, \varepsilon, \omega)$ decays or explodes with $\varepsilon$ (see 1.2 for the definitions). 


\section{A Sketch of the proof of Proposition 3.2}

We recall that $\Omega_{2}:=(0, \pi)^{2}, \omega$ is any open domain contained in $\Omega_{2}, z$ is the solution of 1.7 (in $(0, \tilde{T})$ instead of in $(0, T))$ and the weights are given in 3.1. In addition, in this section $Q:=(0, \tilde{T}) \times \Omega_{2}$, $Q_{\omega}:=(0, \tilde{T}) \times \omega, \Sigma_{l, r}:=(0, \tilde{T}) \times\{0, \pi\} \times(0, \pi)$ and $\Sigma_{b, t}:=(0, \tilde{T}) \times(0, \pi) \times\{0, \pi\}$.

We first remark that the auxiliary functions $\eta^{ \pm}$satisfy:

$$
\begin{array}{r}
\eta^{+}=\eta^{-}, \quad \partial_{n} \eta^{+}+\partial_{n} \eta^{-}=0, \quad\left|\nabla \eta^{+}\right|=\left|\nabla \eta^{-}\right| \text {on }\{0, \pi\} \times(0, \pi), \\
\partial_{n} \eta^{ \pm}<0 \text { on }(0, \pi) \times\{0, \pi\} .
\end{array}
$$

In addition, there is $\delta>0$ a constant that just depends on the control domain and fixed for this section such that:

$$
\left|\nabla \eta^{ \pm}\right| \geq \delta>0 \text { in } \overline{\Omega_{2}} \backslash \omega_{0} .
$$

As for the weights, we have the usual bounds (see Remark 3.1):

$$
\left|\partial_{x_{i}} \alpha_{ \pm}\right|=\left|\partial_{x_{i}} \xi_{ \pm}\right| \leq C \lambda \xi_{ \pm}, \quad\left|\partial_{t} \alpha_{ \pm}\right| \leq C \tilde{T} \xi_{ \pm}^{2}, \quad\left|\partial_{t^{2}}^{2} \alpha_{ \pm}\right| \leq C \xi_{ \pm}^{2}\left(1+\tilde{T}^{2} \xi_{ \pm}\right) .
$$

In order to prove the Carleman inequality, as explained above, there is nothing original in the proof, since we do a mixture of the scheme of [20, Proposition 1] and [12, Lemma1]. In particular, we also consider the change of variables:

$$
\psi^{ \pm}:=e^{-s \alpha_{ \pm}} z
$$

We remark that:

$$
\alpha_{+}=\alpha_{-}, \quad \xi_{+}=\xi_{-}, \quad \psi^{+}=\psi^{-} \text {on } \Sigma_{l r} .
$$

Moreover, $\psi$ has Dirichlet boundary conditions on $\Sigma_{b t}$. As for $\Sigma_{l r}$, since $z$ has Neumann boundary conditions we have the equality:

$$
\partial_{n} \psi^{ \pm}=s \lambda \xi_{ \pm} \partial_{n} \eta^{ \pm} \psi^{ \pm} .
$$

In addition, combining $(A .1)_{1}, A .5$ and $(A .6)$, we have the equality:

$$
\left|\nabla \psi^{+}\right|=\left|\nabla \psi^{-}\right| \text {on } \Sigma_{l r} .
$$

We finally remark that:

$$
L_{1}^{ \pm} \psi^{ \pm}+L_{2}^{ \pm} \psi^{ \pm}=L_{3}^{ \pm} \psi^{ \pm},
$$

for:

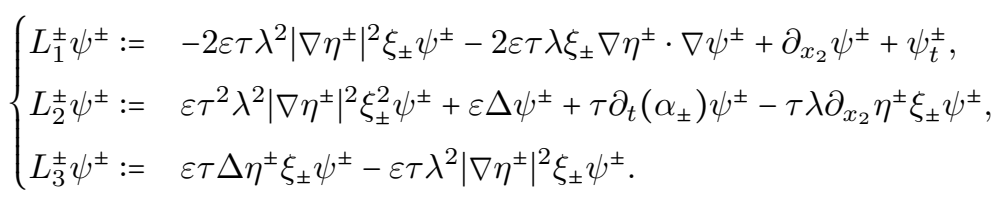

As usual, we denote $\left(L_{i}^{ \pm} \psi\right)_{j}$ the $j$-th term of $L_{i}^{ \pm} \psi$ and calculate the product $\left\langle L_{1}^{ \pm} \psi^{ \pm}, L_{2}^{ \pm} \psi^{ \pm}\right\rangle_{L^{2}(Q)}$. Most of the operations here are repetitions of [20, Proposition 1] with the exception of what we do with the boundary term when integrating by parts (and with the difference that $z$ is a solution of the direct equation in 20]). Thus, we skip some operations. 
To begin with, we have for $\lambda \geq C$ and $\tau \geq C \tilde{T}^{2}$ (see A.2 for the definition of $\delta$ ) the estimate:

$$
\begin{aligned}
& \left\langle\left(L_{1}^{ \pm} \psi^{ \pm}\right)_{1}+\left(L_{1}^{ \pm} \psi^{ \pm}\right)_{2},\left(L_{2}^{ \pm} \psi^{ \pm}\right)_{1}\right\rangle_{L^{2}(Q)} \\
& =\varepsilon^{2} \tau^{3} \lambda^{4} \iint_{Q}\left|\nabla \eta^{ \pm}\right|^{4} \xi_{ \pm}^{3}\left|\psi^{ \pm}\right|^{2} d x d t+O\left(\varepsilon^{2} s^{2} \lambda^{3} \iint_{Q} \xi_{ \pm}^{3}\left|\psi^{ \pm}\right|^{2} d x d t\right)-\varepsilon^{2} \tau^{3} \lambda^{3} \iint_{\Sigma_{l r}}\left|\nabla \eta^{ \pm}\right|^{2} \xi_{ \pm}^{3} \partial_{n} \eta^{ \pm}\left|\psi^{ \pm}\right|^{2} d x d t \\
& \quad \geq \frac{3 \delta^{4}}{4} \varepsilon^{2} \tau^{3} \lambda^{4} \iint_{Q} \xi_{ \pm}^{3}\left|\psi^{ \pm}\right|^{2} d x d t-\delta^{4} \varepsilon^{2} \tau^{3} \lambda^{4} \iint_{Q_{\omega_{0}}} \xi_{ \pm}^{3}\left|\psi^{ \pm}\right|^{2} d x d t-\varepsilon^{2} \tau^{3} \lambda^{3} \iint_{\Sigma_{l r}}\left|\nabla \eta^{ \pm}\right|^{2} \xi_{ \pm}^{3} \partial_{n} \eta^{ \pm}\left|\psi^{ \pm}\right|^{2} d x d t .
\end{aligned}
$$

Moreover, after an integration by parts, considering that $\psi^{ \pm}=0$ on $\Sigma_{b t}$ and A.3), we obtain for $\lambda \geq 1$ and $\tau \geq C\left(\tilde{T}+\tilde{T}^{2}\right)$ the bound:

$$
\left\langle\left(L_{1}^{ \pm} \psi^{ \pm}\right)_{3}+\left(L_{1}^{ \pm} \psi^{ \pm}\right)_{4},\left(L_{2}^{ \pm} \psi^{ \pm}\right)_{1}\right\rangle_{L^{2}(Q)}=O\left(\varepsilon \tau^{2} \lambda^{3} \iint_{Q} \xi_{ \pm}^{2}\left|\psi^{ \pm}\right|^{2} d x d t+\varepsilon \tilde{T} \tau^{2} \lambda^{2} \iint_{Q} \xi_{ \pm}^{3}\left|\psi^{ \pm}\right|^{2} d x d t\right) .
$$

Summing up, thanks to A.10, A.11, A.1, A.2 and A.5, we have for $\lambda \geq C$ and $\tau \geq C\left(\tilde{T}+\tilde{T}^{2}\right) \varepsilon^{-1}$ :

$$
\sum_{i \in\{+,-\}}\left\langle L_{1}^{i} \psi^{i},\left(L_{2}^{i} \psi^{i}\right)_{1}\right\rangle_{L^{2}(Q)} \geq \sum_{i \in\{+,-\}} \frac{\delta^{4}}{2} \varepsilon^{2} \tau^{3} \lambda^{4} \iint_{Q} \xi_{i}^{3}\left|\psi^{i}\right|^{2} d x d t-\delta^{4} \varepsilon^{2} \tau^{3} \lambda^{4} \iint_{Q_{\omega_{0}}} \xi_{i}^{3}\left|\psi^{i}\right|^{2} d x d t .
$$

To continue with, we have that, integrating by parts, with Cauchy-Schwarz inequality and by (A.1):

$$
\begin{aligned}
\left\langle\left(L_{1}^{ \pm} \psi^{ \pm}\right)_{1},\left(L_{2}^{ \pm} \psi^{ \pm}\right)_{2}\right\rangle_{L^{2}(Q)}=2 \varepsilon^{2} \tau \lambda^{2} \iint_{Q}\left|\nabla \eta^{ \pm}\right|^{2} \xi_{ \pm}\left|\nabla \psi^{ \pm}\right|^{2} d x d t-2 \varepsilon^{2} \tau \lambda^{2} \iint_{\Sigma_{l r}}\left|\nabla \eta^{ \pm}\right|^{2} \xi_{ \pm} \partial_{n} \psi^{ \pm} \psi^{ \pm} d x_{2} d t \\
+O\left(\varepsilon^{2} \tau^{2} \lambda^{4} \iint_{Q} \xi_{ \pm}^{2}\left|\psi^{ \pm}\right|^{2} d x d t+\varepsilon^{2} \iint_{Q}\left(\tau \xi+\lambda^{2}\right)\left|\nabla \psi^{ \pm}\right|^{2} d x d t\right)
\end{aligned}
$$

Next, we have again by integration by parts that:

$$
\begin{aligned}
& \left\langle\left(L_{1}^{ \pm} \psi^{ \pm}\right)_{2},\left(L_{2}^{ \pm} \psi^{ \pm}\right)_{2}\right\rangle_{L^{2}(Q)} \\
= & -2 \varepsilon^{2} \tau \lambda \iint_{\Sigma_{b t}} \partial_{n} \eta^{ \pm} \xi_{ \pm}\left|\partial_{n} \psi^{ \pm}\right|^{2} d x_{1} d t-2 \varepsilon^{2} \tau \lambda \iint_{\Sigma_{l r}}\left(\partial_{\mathrm{tg}} \eta^{ \pm} \partial_{\mathrm{tg}} \psi^{ \pm}+\partial_{n} \eta^{ \pm} \partial_{n} \psi^{ \pm}\right) \xi_{ \pm} \partial_{n} \psi^{ \pm} d x_{2} d t \\
+ & O\left(\varepsilon^{2} \tau \lambda \iint_{Q} \xi_{ \pm}\left|\nabla \psi^{ \pm}\right|^{2} d x d t\right)+2 \varepsilon^{2} \tau \lambda^{2} \iint_{Q} \xi_{ \pm}\left|\nabla \eta^{ \pm} \cdot \nabla \psi^{ \pm}\right|^{2} d x d t+\varepsilon^{2} \tau \lambda \iint_{Q} \xi_{ \pm} \nabla \eta^{ \pm} \cdot \nabla\left|\nabla \psi^{ \pm}\right|^{2} d x d t
\end{aligned}
$$

As for the term of the gradient, we have the equality:

$$
\begin{array}{r}
\varepsilon^{2} \tau \lambda \iint_{Q} \xi_{ \pm} \nabla \eta^{ \pm} \cdot \nabla\left|\nabla \psi^{ \pm}\right|^{2} d x d t \\
=-\varepsilon^{2} \tau \lambda^{2} \iint_{Q}\left|\nabla \eta^{ \pm}\right|^{2} \xi_{ \pm}\left|\nabla \psi^{ \pm}\right|^{2} d x d y+O\left(\varepsilon^{2} \tau \lambda \iint_{Q} \xi_{ \pm}\left|\nabla \psi^{ \pm}\right|^{2} d x d t\right) \\
+\varepsilon^{2} \tau \lambda \iint_{\Sigma_{b t}} \partial_{n} \eta^{ \pm} \xi_{ \pm}\left|\partial_{n} \psi^{ \pm}\right|^{2} d x_{1} d t+\varepsilon^{2} \tau \lambda \iint_{\Sigma_{l r}} \partial_{n} \eta^{ \pm} \xi_{ \pm}\left|\nabla \psi^{ \pm}\right|^{2} d x_{2} d t
\end{array}
$$

Next, we have that:

$$
\left\langle\left(L_{1}^{ \pm} \psi^{ \pm}\right)_{3},\left(L_{2}^{ \pm} \psi^{ \pm}\right)_{2}\right\rangle_{L^{2}(Q)}=O\left(\varepsilon \iint_{\Sigma_{b t}}\left|\partial_{n} \psi^{ \pm}\right|^{2} d x_{1} d t\right)+\varepsilon \iint_{\Sigma_{l r}} \partial_{x_{2}} \psi^{ \pm} \partial_{n} \psi^{ \pm} d x_{2} d t .
$$

To continue with, we have the equality:

$$
\left\langle\left(L_{1}^{ \pm} \psi^{ \pm}\right)_{4},\left(L_{2}^{ \pm} \psi^{ \pm}\right)_{2}\right\rangle_{L^{2}(Q)}=\varepsilon \iint_{\Sigma_{l r}} \psi_{t}^{ \pm} \partial_{n} \psi^{ \pm} d x_{2} d t .
$$

So, before adding up, because of $A .1{ }_{2}$, we have for $\lambda \geq C$ and $\tau \geq C\left(\tilde{T}+\tilde{T}^{2}\right) \varepsilon^{-1}$ the bound:

$$
-2 \varepsilon^{2} \tau \lambda \iint_{\Sigma_{b t}} \partial_{n} \eta^{ \pm} \xi_{ \pm}\left|\partial_{n} \psi^{ \pm}\right|^{2} d x_{1} d t+\varepsilon^{2} \tau \lambda \iint_{\Sigma_{b t}} \partial_{n} \eta^{ \pm} \xi_{ \pm}\left|\partial_{n} \psi^{ \pm}\right|^{2} d x_{1} d t+O\left(\varepsilon \iint_{\Sigma_{b t}}\left|\partial_{n} \psi^{ \pm}\right|^{2} d x_{1} d t\right) \geq 0
$$


Summing up, if we consider (A.12)-A.18, , A.1), A.2) and (A.5), we get that:

$$
\begin{aligned}
\sum_{i \in\{+,-\}}\left\langle L_{1}^{i} \psi^{i},\left(L_{2}^{i} \psi^{i}\right)_{2}\right\rangle_{L^{2}(Q)} \geq & \sum_{i \in\{+,-\}} O\left(\varepsilon^{2} \tau^{2} \lambda^{4} \iint_{Q} \xi_{i}^{2}\left|\psi^{i}\right|^{2} d x d t\right) \\
& +\sum_{i \in\{+,-\}} \frac{\delta^{2}}{2} \varepsilon^{2} \tau \lambda^{2} \iint_{Q} \xi_{i}\left|\nabla \psi^{i}\right|^{2} d x d t-\delta^{2} \varepsilon^{2} \tau \lambda^{2} \iint_{Q_{\omega_{0}}} \xi_{i}\left|\nabla \psi^{i}\right|^{2} d x d t .
\end{aligned}
$$

As for the rest of the terms, it is easy to verify for $\lambda \geq 1$ and $\tau \geq C\left(\tilde{T}+\tilde{T}^{2}\right) \varepsilon^{-1}$ the bound:

$$
\sum_{i \in\{+,-\}}\left\langle L_{1}^{i} \psi^{i},\left(L_{2}^{i} \psi^{i}\right)_{3}+\left(L_{2}^{i} \psi^{i}\right)_{4}\right\rangle_{L^{2}(Q)}=\sum_{i \in\{+,-\}} O\left(\varepsilon^{2} \tau^{3} \lambda^{3} \iint_{Q} \xi_{i}^{3}\left|\psi^{i}\right|^{2} d x d t\right)
$$

So, if we add A.13, A.19 and A.20, we get after absorptions for $\lambda \geq C$ and $\tau \geq C\left(\tilde{T}+\tilde{T}^{2}\right) \varepsilon^{-1}$ that:

$$
\begin{aligned}
2 \sum_{i \in\{+,-\}}\left\langle L_{1}^{i} \psi^{i}, L_{2}^{i} \psi^{i}\right\rangle_{L^{2}(Q)}+2 \delta^{4} \varepsilon^{2} \tau^{3} \lambda^{4} & \iint_{Q_{\omega_{0}}} \xi_{i}^{3}\left|\psi^{i}\right|^{2} d x d t+2 \delta^{2} \varepsilon^{2} \tau \lambda^{2} \iint_{Q_{\omega_{0}}} \xi_{i}\left|\nabla \psi^{i}\right|^{2} d x d t \\
& \geq \sum_{i \in\{+,-\}} \frac{\delta^{4}}{2} \varepsilon^{2} \tau^{3} \lambda^{4} \iint_{Q} \xi_{i}^{3}\left|\psi^{i}\right|^{2} d x d t+\frac{\delta^{2}}{2} \varepsilon^{2} \tau \lambda^{2} \iint_{Q} \xi_{i}\left|\nabla \psi^{i}\right|^{2} d x d t .
\end{aligned}
$$

So, considering A.8 and $A .9 y_{3}$ we find after some easy absorptions for $\lambda \geq C$ and $\tau \geq C\left(\tilde{T}+\tilde{T}^{2}\right) \varepsilon^{-1}$ the estimate:

$$
\begin{aligned}
\sum_{i \in\{+,-\}}\left\|L_{1}^{i} \psi^{i}\right\|_{L^{2}(Q)}^{2}+\left\|L_{1}^{i} \psi^{i}\right\|_{L^{2}(Q)}^{2} & +\frac{\delta^{4}}{4} \varepsilon^{2} \tau^{3} \lambda^{4} \iint_{Q} \xi_{i}^{3}\left|\psi^{i}\right|^{2} d x d t+\frac{\delta^{2}}{2} \varepsilon^{2} \tau \lambda^{2} \iint_{Q} \xi_{i}\left|\nabla \psi^{i}\right|^{2} d x d t \\
& \leq \sum_{i \in\{+,-\}} 2 \delta^{4} \varepsilon^{2} \tau^{3} \lambda^{4} \iint_{Q_{\omega_{0}}} \xi_{i}^{3}\left|\psi^{i}\right|^{2} d x d t+2 \delta^{2} \varepsilon^{2} \tau \lambda^{2} \iint_{Q_{\omega_{0}}} \xi_{i}\left|\nabla \psi^{i}\right|^{2} d x d t .
\end{aligned}
$$

Moreover, if we consider in A.9 the equations of $\Delta \psi^{ \pm}$and $\partial_{t} \psi^{ \pm}$, we have, after usual absorptions, for $\lambda \geq C$ and $\tau \geq C\left(\tilde{T}+\tilde{T}^{2}\right) \varepsilon^{-1}$, the inequality:

$$
\begin{array}{r}
\sum_{i \in\{+,-\}} \tau^{-1} \iint_{Q} \xi^{-1}\left(\varepsilon^{-2}\left|\psi_{t}^{i}\right|^{2}+\left|\Delta \psi^{i}\right|^{2}\right) d x d t+\frac{\delta^{4}}{16} \tau^{3} \lambda^{4} \iint_{Q} \xi_{i}^{3}\left|\psi^{i}\right|^{2} d x d t+\frac{\delta^{2}}{8} \tau \lambda^{2} \iint_{Q} \xi_{i}\left|\nabla \psi^{i}\right|^{2} d x d t \\
\leq \sum_{i \in\{+,-\}} 2 \delta^{4} \tau^{3} \lambda^{4} \iint_{Q_{\omega_{0}}} \xi_{i}^{3}\left|\psi^{i}\right|^{2} d x d t+2 \delta^{2} \tau \lambda^{2} \iint_{Q_{\omega_{0}}} \xi_{i}\left|\nabla \psi^{i}\right|^{2} d x d t .
\end{array}
$$

From (A.21) it is well-known how to obtain 3.2 (see, for instance, 13] and [12]).

\section{References}

[1] F. Boyer and P. Fabrie. Mathematical Tool for the Study of the Incompressible Navier-Stokes Equations and Related Models. Springer, first edition, 2013.

[2] N. Carreño and S. Guerrero. On the non-uniform null controllability of a linear KdV equation. Asymptot. Anal., 94(1-2):33-69, 2015.

[3] N. Carreño and S. Guerrero. Uniform null controllability of a linear KdV equation using two controls. J. Math. Anal. Appl., 457(1):922-943, 2018. 
[4] F. W. Chaves-Silva and G. Lebeau. Spectral inequality and optimal cost of controllability for the Stokes system. ESAIM Control Optim. Calc. Var., 22(4):1137-1162, 2016.

[5] P. Cornilleau and S. Guerrero. Controllability and observability of an artificial advection-diffusion problem. Math. Control Signals Systems, 24(3):265-294, 2012.

[6] P. Cornilleau and S. Guerrero. On the cost of null-control of an artificial advection-diffusion problem. ESAIM Control Optim. Calc. Var., 19(4):1209-1224, 2013.

[7] J.-M. Coron. On the controllability of the 2-D incompressible Navier-Stokes equations with the Navier slip boundary conditions. ESAIM Control Optim. Calc. Var., 1:35-75, 1996.

[8] J.-M. Coron. Control and Nonlinearity. Number 136. American Mathematical Soc., 2007.

[9] J.-M. Coron and S. Guerrero. Singular optimal control: a linear 1-D parabolic-hyperbolic example. Asymptot. Anal., 44(3, 4):237-257, 2005.

[10] J.-M. Coron, F. Marbach, F. Sueur, and P. Zhang. Controllability of the Navier-Stokes equation in a rectangle with a little help of a distributed phantom force. Ann. PDE, 5(2):17, 2019.

[11] S. Ervedoza and E. Zuazua. Sharp observability estimates for heat equations. Arch. Ration. Mech. An., 202(3):975-1017, 2011.

[12] E. Fernández-Cara, M. González-Burgos, S. Guerrero, and J.-P. Puel. Null controllability of the heat equation with boundary Fourier conditions: the linear case. ESAIM Control Optim. Calc. Var., 12(3):442-465, 2006.

[13] A. V. Fursikov and O. Yu. Imanuvilov. Controllability of Evolution Equations. Number 34. Seoul National University, 1996.

[14] O. Glass. A complex-analytic approach to the problem of uniform controllability of a transport equation in the vanishing viscosity limit. J. Funct. Anal., 258(3):852-868, 2010.

[15] O. Glass and S. Guerrero. On the uniform controllability of the Burgers equation. SIAM J. Control Optim., 46(4):1211-1238, 2007.

[16] O. Glass and S. Guerrero. Some exact controllability results for the linear KdV equation and uniform controllability in the zero-dispersion limit. Asymptot. Anal., 60(1-2):61-100, 2008.

[17] O. Glass and S. Guerrero. Uniform controllability of a transport equation in zero diffusion-dispersion limit. Math. Models Methods Appl. Sci., 19(09):1567-1601, 2009.

[18] S. Guerrero. Local exact controllability to the trajectories of the Navier-Stokes system with nonlinear Navier-slip boundary conditions. ESAIM Control Optim. Calc. Var., 12(3):484-544, 2006.

[19] S. Guerrero, O. Yu. Imanuvilov, and J.-P. Puel. A result concerning the global approximate controllability of the Navier-Stokes system in dimension 3. J. Math. Pures Appl., 98(6):689-709, 2012.

[20] S. Guerrero and G. Lebeau. Singular optimal control for a transport-diffusion equation. Comm. Partial Differential Equations, 32(12):1813-1836, 2007. 
[21] S. Guerrero and C. Montoya. Local null controllability of the N-dimensional Navier-Stokes system with nonlinear Navier-slip boundary conditions and $N-1$ scalar controls. J. Math. Pures Appl., 113:37-69, 2018.

[22] G. Lebeau and L. Robbiano. Contrôle exact de l'équation de la chaleur. Comm. Partial Differential Equations, 20(1-2):335-356, 1995.

[23] J.-L. Lions. Contrôlabilité exacte, perturbations et stabilisation de systemes distribués, tome 1, RMA 8, 1988.

[24] J.-L. Lions and E. Zuazua. A generique uniqueness result for the Stokes system and its control theoretical consequences. Partial differential equations and applications: Collected Papers in Honor of Carlo Pucci, 177:221-235, 1996.

[25] P. Lissy. A link between the cost of fast controls for the 1-D heat equation and the uniform controllability of a 1-D transport-diffusion equation. C. R. Math. Acad. Sci. Paris, 2012.

[26] P. Lissy. Explicit lower bounds for the cost of fast controls for some 1-D parabolic or dispersive equations, and a new lower bound concerning the uniform controllability of the 1-D transportdiffusion equation. J. Differential Equations, 259(10):5331-5352, 2015.

[27] D. L. Russell. Controllability and stabilizability theory for linear partial differential equations: recent progress and open questions. Siam Rev., 20(4):639-739, 1978. 